\title{
Control sets for bilinear and affine systems
}

\author{
Fritz Colonius $^{1}$ (D) Alexandre J. Santana ${ }^{2}$. Juliana Setti ${ }^{2}$
}

Received: 3 June 2021 / Accepted: 25 October 2021 / Published online: 12 November 2021

(c) The Author(s) 2021

\section{Abstract}

For homogeneous bilinear control systems, the control sets are characterized using a Lie algebra rank condition for the induced systems on projective space. This is based on a classical Diophantine approximation result. For affine control systems, the control sets around the equilibria for constant controls are characterized with particular attention to the question when the control sets are unbounded.

Keywords Affine control systems · Bilinear control systems · Control sets · Diophantine approximations

Mathematics Subject Classification 93B05 $\cdot 34 \mathrm{H} 05 \cdot 11 \mathrm{D} 04$

\section{Introduction}

We will study controllability properties of affine control systems of the form

$$
\dot{x}(t)=A x(t)+\sum_{i=1}^{m} u_{i}(t)\left(B_{i} x(t)+c_{i}\right)+d,
$$

where $A, B_{1}, \ldots, B_{m} \in \mathbb{R}^{n \times n}$ and $c_{1}, \ldots, c_{m}, d$ are vectors in $\mathbb{R}^{n}$. The controls $u=\left(u_{1}, \ldots, u_{m}\right)$ have values in a set $\Omega \subset \mathbb{R}^{m}$. The set of admissible controls is $\mathcal{U}=\left\{u \in L^{\infty}\left(\mathbb{R}, \mathbb{R}^{m}\right) \mid u(t) \in \Omega\right.$ for almost all $\left.t\right\}$ or the set $\mathcal{U}_{p c}$ of all piecewise constant functions defined on $\mathbb{R}$ with values in $\Omega$.

Controllability properties of bilinear and affine control systems have been intensely studied in the last 50 years. The classical monograph by Mohler [20] contains sufficient conditions for complete controllability and many applications of bilinear control

$凶 \quad$ Fritz Colonius

fritz.colonius@math.uni-augsburg.de

1 Institut für Mathematik, Universität Augsburg, Augsburg, Germany

2 Departamento de Matemática, Universidade Estadual de Maringá, Maringá, Brazil 
systems. The monograph Elliott [13] emphasizes the use of matrix Lie groups and Lie semigroups and contains a wealth of results on the control of bilinear control systems.

Motivated by the Kalman criterion for controllability of linear systems, an early goal was show that controllability of bilinear control systems (without control restrictions) has an algebraic characterization. This hope did not bear out, in spite of many partial results. The present paper is mainly concerned with the analysis of control sets, that is, maximal subsets of complete approximate controllability in $\mathbb{R}^{n}$, cf. Definition 2.1 and Colonius and Kliemann [10] for a general theory.

Concerning the literature on controllability properties of affine and bilinear systems, many contributions are based on their analysis via the theory of semigroups in Lie groups, this includes Boothby and Wilson [5], Bonnard [3], Jurdjevic and Kupka [16], Gauthier and Bornard [15], Bonnard et al. [4], Jurdjevic and Sallet [17], San Martin [23].

The main result of Do Rocio et al. [12, Theorem 1.3] concerns a connected semigroup $S$ with nonvoid interior in an affine group $G=B \rtimes V$, where $V$ is a finite dimensional vector space and $B$ is a semisimple Lie group that acts transitively on $V \backslash\{0\}$. If the linear action of the canonical projection $\pi(S)$ on $B$ is transitive on $V \backslash\{0\}$, then the affine action of $S$ on $V$ is transitive. This improves an earlier result in [17]. An application to an affine control system of the form

$$
\dot{x}=A x+a+u B x+u b \text { with } u \in \mathbb{R},
$$

where $A, B \in \mathfrak{s l}(2, \mathbb{R})$ and $a, b \in \mathbb{R}^{2}$, results in a sufficient controllability criterion in terms of these parameters.

Answering a question by Sachkov [22], Do Rocio et al. [11] prove that systems of the form (1.2) with $a=b=0$ and unrestricted control may not be completely controllable on $\mathbb{R}^{n} \backslash\{0\}$ while there is no nontrivial proper closed convex cone in $\mathbb{R}^{n}$ which is positively invariant. For the relation to the results in the present paper see Remark 3.17 and also Proposition 5.14.

Our results on control sets will also yield some results on controllability on $\mathbb{R}^{n}$. We do not restrict our attention to the situation where the system semigroup has nonvoid interior in the system group. Correspondingly, our main results are not based on methods for semigroups in Lie groups.

In the first part of this paper we discuss control sets for homogeneous bilinear systems which are a special case of (1.1) with $c_{1}=\cdots=c_{m}=d=0$. It is well known that, for this class of systems, one can separate controllability properties into properties concerning the angular part on the unit sphere $\mathbb{S}^{n-1}$ and the radial part. In particular, by Bacciotti and Vivalda [2, Theorem 1] the induced system on projective space $\mathbb{P}^{n-1}$ is controllable if and only if the induced system on $\mathbb{S}^{n-1}$ is controllable.

Theorem 3.2 shows that every control set $\mathbb{S}_{\mathbb{S}} D$ with nonvoid interior on $\mathbb{S}^{n-1}$ induces a control set $D$ on $\mathbb{R}^{n} \backslash\{0\}$ given by the cone generated by $\mathbb{S} D$ provided that exponential growth and decay can be achieved. Here we use a classical result on Diophantine approximations which allows us to require only the accessibility rank condition on $\mathbb{S}^{n-1}$ in the interior of ${ }_{\mathbb{S}} D$. This result is illustrated by two-dimensional examples. For systems satisfying the accessibility rank condition on projective space, the control sets on the unit sphere and on $\mathbb{R}^{n} \backslash\{0\}$ are characterized in Theorem 3.12 and Theorem 
3.15 , respectively. We remark that under the accessibility rank condition in $\mathbb{R}^{2}$, a complete description of the control sets and of controllability is given in Ayala et al. [1]. Corollary 3.21 characterizes controllability on $\mathbb{R}^{n} \backslash\{0\}$ for systems satisfying only the accessibility rank condition on $\mathbb{P}^{n-1}$ using a recent result by Cannarsa and Sigalotti [7, Theorem 1] which shows that here approximate controllability implies controllability.

In the second part we analyze control sets for general affine systems and their relation to equilibria. If the systems linearized about equilibria are controllable, Theorem 5.6 shows that any pathwise connected set of equilibria is contained in a control set. Additional assumptions on spectral properties of the matrices $A(u)=$ $A+\sum_{i=1}^{m} u_{i} B_{i}, u \in \Omega$, allow us to get more detailed information. In particular, if 0 is an eigenvalue of $A\left(u^{0}\right)$ for some $u^{0} \in \Omega$, one finds an unbounded control set, cf. Theorem 5.13. The main open problem for control sets of affine systems is, if every control set contains an equilibrium.

The contents of this paper are as follows. Section 2 describes basic properties of nonlinear control systems and control sets as well as some notation for bilinear and affine control systems. Section 3 discusses homogeneous bilinear control systems using their projection to the unit sphere. Section 4 briefly describes equilibria of affine systems and Sect. 5 presents results on control sets around such equilibria.

\section{Preliminaries}

In this section we introduce some terminology and notations for control-affine systems and discuss special cases of affine control systems.

\subsection{Control sets}

Control-affine systems on a smooth manifold $M$ have the form

$$
\begin{aligned}
\dot{x}(t) & =f_{0}(x(t))+\sum_{i=1}^{m} u_{i}(t) f_{i}(x(t)), \\
u & \in \mathcal{U}:=\left\{u \in L^{\infty}\left(\mathbb{R}, \mathbb{R}^{m}\right) \mid u(t) \in \Omega \text { for almost all } t \in \mathbb{R}\right\},
\end{aligned}
$$

where $f_{0}, f_{1}, \ldots, f_{m}$ are smooth vector fields on $M$ and the control range $\Omega \subset \mathbb{R}^{m}$ is compact with $0 \in \operatorname{int}(\Omega)$. We assume that for every initial state $x \in M$ and every control function $u \in \mathcal{U}$ there exists a unique solution $\varphi(t, x, u), t \in \mathbb{R}$, satisfying $\varphi(0, x, u)=x$ of (2.1) depending continuously on $x$. The system with $u \equiv 0$ given by

$$
\dot{x}(t)=f_{0}(x(t))
$$

is called the uncontrolled system. It generates a continuous flow $\varphi_{t}$ on $M$. For the general theory of nonlinear control systems we refer to Sontag [24] and Jurdjevic [18]. 
The set of points reachable from $x \in M$ and controllable to $x \in M$ up to time $T>0$ are defined by

$$
\begin{aligned}
& \mathcal{O}_{\leq T}^{+}(x):=\{y \in M \mid \text { there are } 0 \leq t \leq T \text { and } u \in \mathcal{U} \text { with } y=\varphi(t, x, u)\}, \\
& \mathcal{O}_{\leq T}^{-}(x):=\{y \in M \mid \text { there are } 0 \leq t \leq T \text { and } u \in \mathcal{U} \text { with } x=\varphi(t, y, u)\},
\end{aligned}
$$

resp. Furthermore, the reachable set (or "positive orbit") from $x$ and the set controllable to $x$ (or "negative orbit" of $x$ ) are

$$
\mathcal{O}^{+}(x)=\bigcup_{T>0} O_{\leq T}^{+}(x), \quad \mathcal{O}^{-}(x)=\bigcup_{T>0} O_{\leq T}^{-}(x),
$$

resp. The system is called locally accessible in $x$, if $\mathcal{O}_{\leq T}^{+}(x)$ and $\mathcal{O}_{\leq T}^{-}(x)$ have nonvoid interior for all $T>0$ and the system is called locally accessible if this holds in every point $x \in M$. This is guaranteed by the following accessibility rank condition

$$
\operatorname{dim} \mathcal{L} \mathcal{A}\left\{f_{0}, f_{1}, \ldots, f_{m}\right\}(x)=\operatorname{dim} M \text { for all } x \in M
$$

here $\mathcal{L} \mathcal{A}\left\{f_{0}, f_{1}, \ldots, f_{m}\right\}(x)$ is the subspace of the tangent space $T_{x} M$ corresponding to the vector fields, evaluated in $x$, in the Lie algebra generated by $f_{0}, f_{1}, \ldots, f_{m}$.

The trajectories for the convex hull of $\Omega$ can be uniformly approximated on bounded intervals by the trajectories for $\Omega$. Furthermore, trajectories for controls in $\mathcal{U}$ can be uniformly approximated on bounded intervals by trajectories for piecewise constant controls in $\mathcal{U}_{p c}$.

The following definition introduces subsets of complete approximate controllability which are of primary interest in the present paper.

Definition 2.1 A nonvoid set $D \subset M$ is called a control set of system (2.1) if it has the following properties: (i) for all $x \in D$ there is a control function $u \in \mathcal{U}$ such that $\varphi(t, x, u) \in D$ for all $t \geq 0$, (ii) for all $x \in D$ one has $D \subset \overline{\mathcal{O}^{+}(x)}$, and (iii) $D$ is maximal with these properties, that is, if $D^{\prime} \supset D$ satisfies conditions (i) and (ii), then $D^{\prime}=D$.

A control set $D \subset M$ is called an invariant control set if $\bar{D}=\overline{\mathcal{O}^{+}(x)}$ for all $x \in D$. All other control sets are called variant.

If the intersection of two control sets is nonvoid, the maximality property (ii) implies that they coincide. If the system is locally accessible in all $x \in \operatorname{int}(D)$, then int $(D) \subset$ $\mathcal{O}^{+}(x)$ for all $x \in D$ and $D=\mathcal{O}^{-}(x) \cap \overline{\mathcal{O}^{+}(x)}$ for every $x \in$ int $(D)$. The control sets with nonvoid interior for piecewise constant controls in $\mathcal{U}_{p c}$ coincide with those for controls in $\mathcal{U}$. For these and further properties of control sets, we refer to Colonius and Kliemann [10, Chapters 3 and 4].

The following lemma shows that the controllable set of system (1.1) coincides with the reachable set of the time reversed system.

Lemma 2.2 Consider together with system (2.1) the time reversed system

$$
\dot{x}(t)=-f_{0}(x(t))-\sum_{i=1}^{m} v_{i}(t) f_{i}(x(t)), \quad v \in \mathcal{U}
$$


We denote by $\mathcal{O}_{1}^{+}(x)$ and $\mathcal{O}_{1}^{-}(x)$ the reachable set from $x$ and the controllable set to $x$, determined by the system (2.1), respectively, and by $\mathcal{O}_{2}^{+}(x)$ and $\mathrm{O}_{2}^{-}(x)$ the reachable set from $x$ and the controllable set to $x$, determined by the system (2.4), respectively. Then $\mathcal{O}_{1}^{+}(x)=\mathcal{O}_{2}^{-}(x)$ and $\mathcal{O}_{1}^{-}(x)=\mathcal{O}_{2}^{+}(x)$.

Proof For $y=\varphi(T, x, u) \in \mathcal{O}_{1}^{+}(x)$, the absolutely continuous function $\psi(t):=$ $\varphi(T-t, x, u(T-\cdot)), t \in[0, T]$, satisfies $\psi(0)=y, \psi(T)=x$. It is a solution of (2.4) with $v(t):=u(T-t), t \in[0, T]$, since for almost all $t \in[0, T]$

$$
\begin{aligned}
\dot{\psi}(t) & =\frac{d}{d t} \varphi(T-t, y, u(T-\cdot)) \\
& =-f_{0}(\varphi(T-t, y, u(T-\cdot)))-\sum_{i=1}^{m} u_{i}(T-t) f_{i}(\varphi(T-t, y, u(T-\cdot)) \\
& =-f_{0}(\psi(t))-\sum_{i=1}^{m} v_{i}(t) f_{i}(\psi(t)) .
\end{aligned}
$$

Thus $\mathcal{O}_{1}^{+}(x) \subset \mathcal{O}_{2}^{-}(x)$. The other inclusions follow analogously.

\subsection{Affine and bilinear control systems}

Frequently, we abbreviate

$$
A(u):=A+\sum_{i=1}^{m} u_{i} B_{i} \text { for } u \in \Omega \text { and } C:=\left(c_{1}, \ldots, c_{m}\right) \in \mathbb{R}^{n \times m},
$$

hence the columns of $C$ are given by the $c_{i}$. Then (1.1) can be written as

$$
\dot{x}(t)=A(u(t)) x(t)+C u(t)+d .
$$

A special case are bilinear control systems obtained for $d=0$, i.e.

$$
\dot{x}(t)=A x(t)+\sum_{i=1}^{m} u_{i}(t)\left(B_{i} x(t)+c_{i}\right)=A(u(t)) x(t)+C u(t),
$$

and homogeneous bilinear systems of the form

$$
\dot{x}(t)=A x(t)+\sum_{i=1}^{m} u_{i}(t) B_{i} x(t)=A(u(t)) x(t) .
$$

For fixed control $u \in \mathcal{U}$ (1.1) is a nonautonomous inhomogeneous linear differential equation. Denote by $\Phi_{u}(t, s) \in \mathbb{R}^{n \times n}$ the principal matrix solution, i.e., the solution of 


$$
\frac{d}{d t} \Phi_{u}(t, s)=A(u(t)) \Phi_{u}(t, s), \quad \Phi_{u}(s, s)=I .
$$

The solutions $\varphi\left(t, x_{0}, u\right), t \in \mathbb{R}$, of (1.1) with initial condition $\varphi\left(0, x_{0}, u\right)=x_{0} \in \mathbb{R}^{n}$ are given by

$$
\varphi\left(t, x_{0}, u\right)=\Phi_{u}(t, 0) x_{0}+\int_{0}^{t} \Phi_{u}(t, s)[C u(s)+d] d s, \quad t \in \mathbb{R},
$$

and, in particular, the solutions of (2.7) are

$$
\varphi\left(t, x_{0}, u\right)=\Phi_{u}(t, 0) x_{0}, \quad t \in \mathbb{R}
$$

This readily implies for $\alpha \in \mathbb{R}$

$$
\varphi\left(t, \alpha x_{0}, u\right)=\Phi_{u}(t, 0) \alpha x_{0}=\alpha \varphi\left(t, x_{0}, u\right)
$$

\section{Control sets for homogeneous bilinear systems}

We consider homogeneous bilinear control systems of the form (2.7) and describe their control sets.

Since for fixed control $u$, the corresponding differential equations are homogeneous, their controllability properties can often be split into controllability properties for the angles and the radii separately; cf., e.g., Colonius and Kliemann [10, Chapter 7]. Denote the projection of $\mathbb{R}^{n}$ to the Euclidean unit sphere $\mathbb{S}^{n-1}$ by $\pi$ and the projection to real projective space $\mathbb{P}^{n-1}$ (obtained by identifying opposite points on the sphere) by $\mathbb{P}$. For a trajectory of (2.7) define

$$
s(t):=\pi(x(t))=\frac{x(t)}{\|x(t)\|}, \quad t \in \mathbb{R} .
$$

The projected trajectories are trajectories of control-affine systems on $\mathbb{S}^{n-1}$ given by

$$
\begin{aligned}
\dot{s}(t) & =h(u(t), s(t))=h_{0}(s(t))+\sum_{i=1}^{m} u_{i}(t) h_{i}(s(t)), \\
h_{0}(s) & =A s-s^{\top} A s \cdot s, \quad h_{i}(s)=B_{i} s-s^{\top} B_{i} s \cdot s \text { for } i=1, \ldots, m .
\end{aligned}
$$

The vector fields of the system on $\mathbb{S}^{n-1}$ are obtained by subtracting the radial component. The solutions will be denoted by $s\left(t, s_{0}, u\right), t \in \mathbb{R}$. One also obtains an induced control system on projective space $\mathbb{P}^{n-1}$ with vector fields $\mathbb{P} h(u, \cdot)$ since $h_{i}(s)=-h_{i}(-s)$ for all $i$.

Since bilinear control systems as well as their projections to $\mathbb{S}^{n-1}$ and $\mathbb{P}^{n-1}$ are analytic, for these systems, local accessibility is equivalent to the corresponding accessibility rank condition (2.3); cf. Sontag [24, Theorem 12 on p. 179]. 
We note the following simple result showing a first relation between control sets on $\mathbb{R}^{n}$ and control sets on $\mathbb{S}^{n-1}$.

Proposition 3.1 Suppose that $D \subset \mathbb{R}^{n} \backslash\{0\}$ is a control set of system (2.7). Then the projection $\mathbb{P}(D)$ to projective space $\mathbb{P}^{n-1}$ is contained in a control set $\mathbb{P} D$ for the induced system on $\mathbb{P}^{n-1}$, and the projection $\pi(D)$ to the unit sphere $\mathbb{S}^{n-1}$ is contained in a control set $\mathbb{S} D$ for the induced system (3.1) on $\mathbb{S}^{n-1}$. If $D$ has nonvoid interior, then also $\mathbb{P} D$ and $\mathbb{S}_{\mathbb{S}} D$ have nonvoid interiors.

Proof The assertions immediately follow from the definitions and the fact that the projections $\pi$ and $\mathbb{P}$ are open.

Next we will analyze when a control set on the unit sphere $\mathbb{S}^{n-1}$ generates a control set on $\mathbb{R}^{n}$. This result is based on a Diophantine approximation result used for Lemma 3.4 .

Theorem 3.2 Let ${ }_{\mathbb{S}} D$ be a control set with nonvoid interior for the system on the unit sphere $\mathbb{S}^{n-1}$ and suppose that

(i) every point in int $(\mathbb{S} D)$ is locally accessible;

(ii) there are $\alpha_{0}^{+}>1, \delta_{0}>0$, and $\alpha^{-} \in(0,1)$ such that for all $\alpha^{+} \in\left(\alpha_{0}^{+}, \alpha_{0}^{+}+\delta_{0}\right)$ there are points $s^{+}, s^{-} \in$ int $\left({ }_{\mathbb{S}} D\right)$, controls $u^{+}, u^{-} \in \mathcal{U}$, and times $\sigma^{+}, \sigma^{-}>0$ with

$$
\varphi\left(\sigma^{+}, s^{+}, u^{+}\right)=\alpha^{+} s^{+}, \quad \varphi\left(\sigma^{-}, s^{-}, u^{-}\right)=\alpha^{-} s^{-} .
$$

Then the cone $\left\{\alpha s \in \mathbb{R}^{n} \mid \alpha>0, s \in{ }_{\mathbb{S}} D\right\}$ is a control set in $\mathbb{R}^{n}$ with nonvoid interior.

Remark 3.3 The proof of Theorem 3.2 will show that we can replace assumption (ii) by the following assumption:

(ii)' there are $\alpha^{+}>1, \delta_{0} \in(0,1)$, and $\alpha_{0}^{-} \in\left(0,1-\delta_{0}\right)$ such that for all $\alpha^{-} \in$ $\left(\alpha_{0}^{-}, \alpha_{0}^{-}+\delta_{0}\right)$ there are points $s^{+}, s^{-} \in$ int $(\mathbb{S} D)$, controls $u^{+}, u^{-} \in \mathcal{U}$, and times $\sigma^{+}, \sigma^{-}>0$ with (3.2).

Proof First observe that (3.2) implies for the projected system on $\mathbb{S}^{n-1}$

$$
s\left(\sigma^{+}, s^{+}, u^{+}\right)=s^{+}, \quad s\left(\sigma^{-}, s^{-}, u^{-}\right)=s^{-} .
$$

Hence we get periodic solutions in int $\left.{ }_{\mathbb{S}} D\right) \subset \mathbb{S}^{n-1}$.

Step 1: Let $s_{0} \in \operatorname{int}(\mathbb{S} D)$. Then for every $x_{0} \in l:=\left\{\alpha s_{0} \in \mathbb{R}^{n} \mid \alpha>0\right\}$ the closure of the reachable set from $x_{0}$ contains the half-line $l$.

For the proof of this claim, consider arbitrary points $x_{0}=\alpha_{0} s_{0}, x_{1}=\alpha_{1} s_{0} \in l$ with $\alpha_{0}, \alpha_{1}>0$. The strategy is to steer the system from $s_{0}$ to $s^{+}$, then to go $k$ times through the periodic trajectory for $u^{+}$, then to steer the system to $s^{-}$, go $\ell$ times through the periodic trajectory for $u^{-}$, and finally steer the system back to $s_{0}$. The numbers $k, \ell \in \mathbb{N}$ will be adjusted such that the corresponding trajectories in $\mathbb{R}^{n}$ starting in $x_{0}$ approach $x_{1}$. 
By local accessibility in int $\left.{ }_{\mathbb{S}} D\right)$ there are times $\tau_{1}, \tau_{2}, \tau_{3}>0$ and controls $v^{1}, v^{2}$, $v^{3} \in \mathcal{U}$ with

$$
s\left(\tau_{1}, s_{0}, v^{1}\right)=s^{+}, \quad s\left(\tau_{2}, s^{+}, v^{2}\right)=s^{-}, \quad s\left(\tau_{3}, s^{-}, v^{3}\right)=s_{0}
$$

One finds for the system in $\mathbb{R}^{n}$ numbers $\beta_{1}, \beta_{2}, \beta_{3}>0$ with

$$
\begin{aligned}
& \varphi\left(\tau_{1}, x_{0}, v^{1}\right)=\varphi\left(\tau_{1}, \alpha_{0} s_{0}, v^{1}\right)=\beta_{1} s^{+}, \varphi\left(\tau_{2}, s^{+}, v^{2}\right)=\beta_{2} s^{-}, \\
& \varphi\left(\tau_{3}, s^{-}, v^{3}\right)=\beta_{3} s_{0} .
\end{aligned}
$$

Now define for $k, \ell \in \mathbb{N}$ a control function $w^{k, \ell}$ by

$$
\begin{aligned}
w^{k, \ell}(t) & =v^{1}(t) \text { for } t \in\left[0, \tau_{1}\right], \\
w^{k, \ell}(t) & =u^{+}\left(t-\left(\tau_{1}+(i-1) \sigma^{+}\right)\right) \text {for } t \in\left(\tau_{1}+(i-1) \sigma^{+}, \tau_{1}+i \sigma^{+}\right], i=1, \ldots, k, \\
w^{k, \ell}(t) & =v^{2}\left(t-\left(\tau_{1}+k \sigma^{+}\right)\right) \text {for } t \in\left(\tau_{1}+k \sigma^{+}, \tau_{1}+k \sigma^{+}+\tau_{2}\right], \\
w^{k, \ell}(t) & =u^{-}\left(t-\left(\tau_{1}+k \sigma^{+}+\tau_{2}+(i-1) \sigma^{-}\right)\right) \\
\text {for } t & \in\left(\tau_{1}+k \sigma^{+}+\tau_{2}+(i-1) \sigma^{-}, \tau_{1}+k \sigma^{+}+\tau_{2}+i \sigma^{-}\right], \quad i=1, \ldots, \ell, \\
w^{k, \ell}(t) & =v^{3}\left(t-\left(\tau_{1}+k \sigma^{+}+\tau_{2}+\ell \sigma^{-}\right)\right), \\
\text {for } t & \in\left(\tau_{1}+k \sigma^{+}+\tau_{2}+\ell \sigma^{-}, \tau_{1}+k \sigma^{+}+\tau_{2}+\ell \sigma^{-}+\tau_{3}\right] .
\end{aligned}
$$

The corresponding trajectory on $\mathbb{S}^{n-1}$ is periodic and satisfies

$$
\begin{aligned}
s\left(\tau_{1}+i \sigma^{+}, s_{0}, w^{k, \ell}\right) & =s^{+} \text {for } i=0,1, \ldots, k, \\
s\left(\tau_{1}+k \sigma^{+}+\tau_{2}+i \sigma^{-}, s_{0}, w^{k, \ell}\right) & =s^{-} \text {for } i=0,1, \ldots, \ell, \\
s\left(\tau_{1}+k \sigma^{+}+\tau_{2}+\ell \sigma^{-}+\tau_{3}, s_{0}, w^{k, \ell}\right) & =s_{0},
\end{aligned}
$$

and for the corresponding trajectory on $\mathbb{R}^{n}$ one finds using (2.8)

$$
\begin{aligned}
\varphi\left(\tau_{1}+i \sigma^{+}, x_{0}, w^{k, \ell}\right) & =\left(\alpha^{+}\right)^{i} \beta_{1} s^{+} \text {for } i=0,1, \ldots, k, \\
\varphi\left(\tau_{1}+k \sigma^{+}+\tau_{2}+i \sigma^{-}, x_{0}, w^{k, \ell}\right) & =\left(\alpha^{-}\right)^{i} \beta_{2}\left(\alpha^{+}\right)^{k} \beta_{1} s^{-} \text {for } i=0,1, \ldots, \ell, \\
\varphi\left(\tau_{1}+k \sigma^{+}+\tau_{2}+\ell \sigma^{-}+\tau_{3}, x_{0}, w^{k, \ell}\right) & =\beta_{3}\left(\alpha^{-}\right)^{\ell} \beta_{2}\left(\alpha^{+}\right)^{k} \beta_{1} s_{0} .
\end{aligned}
$$

Recall that our goal is to reach $x_{1}=\alpha_{1} s_{0}$ approximately. We apply Lemma 3.4 with $a=\alpha^{+}, b=\left(\alpha^{-}\right)^{-1}$, and $c=\alpha_{1}\left(\beta_{3} \beta_{2} \beta_{1}\right)^{-1}$, where we choose $\alpha^{+} \in\left(\alpha_{0}^{+}, \alpha_{0}^{+}+\delta_{0}\right)$ such that $\frac{\log b}{\log a}=\frac{-\log \alpha^{-}}{\log \alpha^{+}}$is irrational. Thus for every $\varepsilon>0$ there are $k, \ell \in \mathbb{N}$ with

$$
\left|\left(\alpha^{+}\right)^{k}\left(\alpha^{-}\right)^{\ell}-\alpha_{1}\left(\beta_{3} \beta_{2} \beta_{1}\right)^{-1}\right|=\left|\frac{\left(\alpha^{+}\right)^{k}}{\left(\alpha^{-}\right)^{-\ell}}-\alpha_{1}\left(\beta_{3} \beta_{2} \beta_{1}\right)^{-1}\right|<\varepsilon,
$$


hence for all $\varepsilon>0$ there are $k, \ell \in \mathbb{N}$ with

$$
\left|\beta_{3} \beta_{2} \beta_{1}\left(\alpha^{+}\right)^{k}\left(\alpha^{-}\right)^{\ell}-\alpha_{1}\right|<\varepsilon \text {. }
$$

It follows that for some $\delta \in(-\varepsilon, \varepsilon)$ one can choose $k, \ell$ such that

$$
\varphi\left(\tau_{1}+k \sigma^{+}+\tau_{2}+\ell \sigma^{-}+\tau_{3}, x_{0}, w^{k, \ell}\right)=\beta_{3} \beta_{2} \beta_{1}\left(\alpha^{-}\right)^{\ell}\left(\alpha^{+}\right)^{k} s_{0}=\left(\alpha_{1}+\delta\right) s_{0} .
$$

Since $\varepsilon>0$ is arbitrary, it follows that $x_{1}=\alpha_{1} s_{0}$ is in the closure of the reachable set of $x_{0}$ and hence $l$ is contained the closure of the reachable set from $x_{0}$.

Step 2: Let $x_{1}, x_{2} \in\left\{\alpha s \in \mathbb{R}^{n} \mid \alpha>0, s \in{ }_{\mathbb{S}} D\right\}$, hence there are $\alpha_{1}, \alpha_{2}>0$ and $s_{1}, s_{2} \in \mathbb{S}_{\mathbb{S}} D$ with $x_{1}=\alpha_{1} s_{1}$ and $x_{2}=\alpha_{2} s_{2}$. Then there are a control $u_{1}$ and a time $t_{1} \geq 0$ with $s\left(t_{1}, s_{1}, u_{1}\right)=s_{0}$, hence $\varphi\left(t_{1}, x_{1}, u_{1}\right)=\gamma_{1} s_{0} \in l$ for some $\gamma_{1}>0$. Since $s_{0}, s_{2} \in \mathbb{S} D$ one finds, for $\varepsilon>0$, a control $u_{2}$ and a time $t_{2} \geq 0$ such that, for $s_{3}:=s\left(t_{2}, s_{0}, u_{2}\right)$,

$$
\left\|s_{3}-s_{2}\right\|<\varepsilon / \alpha_{2} \text { and }\left\|\alpha_{2} s_{3}-x_{2}\right\|=\left\|\alpha_{2} s_{3}-\alpha_{2} s_{2}\right\|<\varepsilon \text {. }
$$

The trajectory in $\mathbb{R}^{n}$ satisfies $\varphi\left(t_{2}, s_{0}, u_{2}\right)=\gamma_{2} s_{3}$ for some $\gamma_{2}>0$. By (2.8) it follows that

$$
\varphi\left(t_{2}, \frac{\alpha_{2}}{\gamma_{2}} s_{0}, u_{2}\right)=\frac{\alpha_{2}}{\gamma_{2}} \gamma_{2} s_{3}=\alpha_{2} s_{3} .
$$

Step 1 implies that one finds arbitrarily close to $\frac{\alpha_{2}}{\gamma_{2}} s_{0} \in l$ points in the reachable set from $\gamma_{1} s_{0}$, hence in the reachable set from $x_{1}$. By continuous dependence on the initial value, it follows that under the control $u_{2}$ points in the reachable set from $x_{1}$ are steered into the $\varepsilon$-neighborhood of $x_{2}$. Since $\varepsilon>0$ is arbitrary, this shows that $x_{2}$ is in the closure of the reachable set from $x_{1}$.

Step 3: We have shown that the cone $D^{\prime}:=\left\{\alpha s \in \mathbb{R}^{n} \mid \alpha>0, s \in \mathbb{S} D\right\}$ is a set of complete approximate controllability. It is maximal with this property, since any set of approximate controllability in $\mathbb{R}^{n}$ projects to a set of approximate controllability in $\mathbb{S}^{n-1}$, and $\mathbb{S}_{\mathbb{S}} D$ is a maximal set of approximate controllability. Finally, for every point $x \in D^{\prime}$ there is a control $u$ with $\varphi(t, x, u) \in D^{\prime}$ for all $t \geq 0$, since this holds in $\mathbb{S}_{\mathbb{S}} D$. Hence the cone $D^{\prime}$ is a control set and it has a nonvoid interior.

Step 1 in the proof above is based on the following lemma which uses a Diophantine approximation property.

Lemma 3.4 Let $a, b$, c be real numbers with $a, b>1, c>0$, and $\frac{\log b}{\log a} \in \mathbb{R} \backslash \mathbb{Q}$. Then for every $\varepsilon>0$ there are $k, \ell \in \mathbb{N}$ such that $\left|a^{k} b^{-\ell}-c\right|<\varepsilon$.

Proof Since the logarithm is continuously invertible, it suffices to show that for every $\varepsilon>0$ there are $k, \ell \in \mathbb{N}$ with

$$
\varepsilon>\left|\log \left(a^{k} b^{-\ell}\right)-\log c\right|=|k \log a-\ell \log b-\log c|,
$$


or, dividing by $\log a>0$,

$$
\left|k-\ell \frac{\log b}{\log a}-\frac{\log c}{\log a}\right|<\frac{\varepsilon}{\log a} .
$$

We use the following Diophantine approximation result which is due to Tchebychef [25, Théorème, p. 679]: For any irrational number $\alpha$ and any $\beta \in \mathbb{R}$ the inequality $x|y-\alpha x-\beta|<2$ has an infinite number of solutions in $x \in \mathbb{N}, y \in \mathbb{Z}$. Observe that here also $y \in \mathbb{N}$ if $\alpha>0$, since then $\operatorname{sgn}(y)=\operatorname{sgn}(\alpha x)=\operatorname{sgn}(x)=1$. For an application to the problem above, let $\alpha=\frac{\log b}{\log a}>0, \beta=\frac{\log c}{\log a}, x=\ell, y=k$. One obtains that

$$
\ell\left|k-\ell \frac{\log b}{\log a}-\frac{\log c}{\log a}\right|<2
$$

has an infinite number of solutions $k, \ell \in \mathbb{N}$. Choosing $\ell$ large enough such that $\frac{2 \log a}{\ell}<\varepsilon$ and dividing by $\ell$ one gets, as desired,

$$
\left|k-\ell \frac{\log b}{\log a}-\frac{\log c}{\log a}\right|<\frac{2}{\ell}<\frac{\varepsilon}{\log a} .
$$

Remark 3.5 The Diophantine approximation result used above is closely related to a theorem due to Minkowski on inhomogeneous linear Diophantine approximation, cf. Cassels, [8, Theorem I in Chapter III]. Here the existence of integers $x, y$ solving $x|y-\alpha x-\beta|<\frac{1}{4}$ is established, but not the existence of infinitely many pairs $x, y$ with this property, as required for the proof above.

Remark 3.6 Suppose that for a control set ${ }_{\mathbb{S}} D$ on the unit sphere, every point in the interior is locally accessible and there are control values $u^{ \pm} \in$ int $(\Omega)$ such that $A\left(u^{+}\right)$ has an eigenvalue $\lambda^{+}>0$ and $A\left(u^{-}\right)$has an eigenvalue $\lambda^{-}<0$ with eigenspaces satisfying $E\left(\lambda^{ \pm}\right) \cap$ int $(\mathbb{S} D) \neq \varnothing$. Then assumption (ii) of Theorem 3.15 holds. In fact, all points $s^{ \pm} \in E\left(\lambda^{ \pm}\right) \cap$ int $\left({ }_{\mathbb{S}} D\right)$ are equilibria for the induced system on $\mathbb{S}^{n-1}$ with $A\left(u^{ \pm}\right) s^{ \pm}=\lambda^{ \pm} s^{ \pm}$. This implies for all $\sigma^{ \pm}>0$ and the constant controls $u^{ \pm} \in \Omega$ that

$$
\begin{aligned}
& \varphi\left(\sigma^{+}, s^{+}, u^{+}\right)=\alpha_{0}^{+} s^{+} \text {with } \alpha_{0}^{+}:=e^{\lambda^{+} \sigma^{+}}>1, \\
& \varphi\left(\sigma^{-}, s^{-}, u^{-}\right)=\alpha^{-} s^{-} \text {with } \alpha^{-}:=e^{\lambda^{-} \sigma^{-}}<1 .
\end{aligned}
$$

This follows, since the solutions of $\dot{x}=A\left(u^{ \pm}\right) x, x(0)=s^{ \pm}$, are given by

$$
\varphi\left(t, s^{ \pm}, u^{ \pm}\right)=e^{A\left(u^{ \pm}\right) t} s^{ \pm}=e^{\lambda^{ \pm} t} s^{ \pm} .
$$

Varying $\sigma^{+}$, we get that $\varphi\left(\sigma^{+}, s^{+}, u^{+}\right)=\alpha^{+} s^{+}$for all $\alpha^{+} \in\left(\alpha_{0}^{+}, \alpha_{0}^{+}+\delta_{0}\right)$ and some $\delta_{0}>0$. 
The following two examples illustrate Theorem 3.2. We consider problems in $\mathbb{R}^{2}$ where the induced system on the unit circle is not locally accessible. First let $A$ be given in Jordan normal form $A=\left[\begin{array}{cc}\lambda_{1} & 0 \\ 0 & \lambda_{2}\end{array}\right]$ and let the matrices $B_{1}$ and $B_{2}$ be diagonal. The situation is a bit more complicated than in Remark 3.6, since the intersections of the relevant eigenspaces with the unit sphere yield boundary points of the control set $\mathbb{S} D$.

Example 3.7 Consider a system of the form

$$
\left[\begin{array}{l}
\dot{x} \\
\dot{y}
\end{array}\right]=\left(\left[\begin{array}{cc}
\lambda_{1} & 0 \\
0 & \lambda_{2}
\end{array}\right]+u(t)\left[\begin{array}{cc}
b_{11} & 0 \\
0 & b_{21}
\end{array}\right]+v(t)\left[\begin{array}{cc}
b_{12} & 0 \\
0 & b_{22}
\end{array}\right]\right)\left[\begin{array}{l}
x \\
y
\end{array}\right],
$$

with $\lambda_{1}, \lambda_{2} \in \mathbb{R}$ and control values $(u(t), v(t)) \in \Omega \subset \mathbb{R}^{2}$. This can be written as

$$
\left[\begin{array}{c}
\dot{x} \\
\dot{y}
\end{array}\right]=\left[\begin{array}{cc}
\lambda_{1}+b_{11} u+b_{12} v & 0 \\
0 & \lambda_{2}+b_{21} u+b_{22} v
\end{array}\right]\left[\begin{array}{l}
x \\
y
\end{array}\right]=A(u, v)\left[\begin{array}{l}
x \\
y
\end{array}\right] .
$$

For all $(u, v) \in \Omega$ the eigenvalues $\mu_{1}(u, v)=\lambda_{1}+b_{11} u+b_{12} v$ and $\mu_{2}(u, v)=$ $\lambda_{2}+b_{21} u+b_{22} v$ of $A(u, v)$ have the eigenspaces $\mathbb{R} \times\{0\}$ and $\{0\} \times \mathbb{R}$, resp. Assume that there are control values $\left(u_{1}, v_{1}\right),\left(u_{2}, v_{2}\right) \in \Omega$ with

$$
\mu_{1}\left(u_{1}, v_{1}\right)>0, \mu_{2}\left(u_{1}, v_{1}\right)<0 \text { and } \mu_{1}\left(u_{2}, v_{2}\right)<0, \mu_{2}\left(u_{2}, v_{2}\right)>0 .
$$

For $\left(u_{1}, v_{1}\right)$ the eigenspace $\mathbb{R} \times\{0\}$ is attracting and for $\left(u_{2}, v_{2}\right)$ the eigenspace $\{0\} \times \mathbb{R}$ is attracting. One easily verifies that on the unit circle $\mathbb{S}^{1}$ there are four open and invariant control sets $\mathbb{S}_{\mathbb{S}}, i=1, \ldots, 4$, with nonvoid interior on the unit sphere separated by the four points in the intersection of the eigenspaces $\mathbb{R} \times\{0\}$ and $\{0\} \times \mathbb{R}$ with $\mathbb{S}^{1}$. The four points in this intersection are invariant for all $(u, v)$, hence they are not locally accessible, while every point in the control sets is locally accessible.

In order to verify condition (3.2), assume that there is $\left(u_{3}, v_{3}\right) \in \Omega$ with

$$
\mu_{1}\left(u_{3}, v_{3}\right)=0 \text { and } \mu_{2}\left(u_{3}, v_{3}\right)>0
$$

Let $\tau_{1}>0$, and define $\tau_{2}:=\tau_{1} \frac{\mu_{1}\left(u_{1}, v_{1}\right)-\mu_{2}\left(u_{1}, v_{1}\right)}{\mu_{2}\left(u_{3}, v_{3}\right)}>0$ and

$$
\left(u^{+}(t), v^{+}(t)\right):=\left\{\begin{array}{ll}
\left(u_{1}, v_{1}\right) & \text { for } t \in\left[0, \tau_{1}\right] \\
\left(u_{3}, v_{3}\right) & \text { for } t \in\left(\tau_{1}, \tau_{2}+\tau_{1}\right]
\end{array} .\right.
$$

Fix a point $s^{+} \in{ }_{\mathbb{S}} D_{i}$. Then it follows that

$$
\begin{aligned}
\varphi\left(\tau_{2}+\tau_{1}, s^{+}, u^{+}, v^{+}\right) & =\varphi\left(\tau_{2}, \varphi\left(\tau_{1}, s^{+}, u_{1}, v_{1}\right), u_{3}, v_{3}\right) \\
& =\left[\begin{array}{cc}
e^{0} & 0 \\
0 & e^{\tau_{2} \mu_{2}\left(u_{3}, v_{3}\right)}
\end{array}\right]\left[\begin{array}{c}
e^{\tau_{1} \mu_{1}\left(u_{1}, v_{1}\right)} \\
e^{\tau_{1} \mu_{2}\left(u_{1}, v_{1}\right)}
\end{array}\right] s^{+}=e^{\tau_{1} \mu_{1}\left(u_{1}, v_{1}\right)} s^{+}
\end{aligned}
$$


Since $\tau_{1}>0$ is arbitrary, the first equality in (3.2) holds with $\sigma^{+}=\tau_{2}+\tau_{1}$ and $\alpha^{+}=e^{\tau_{1} \mu_{1}\left(u_{1}, v_{1}\right)}>1$.

Analogously, fix a point $s^{-} \in{ }_{\mathbb{S}} D_{i}$. Assume that there is $\left(u_{4}, v_{4}\right) \in \Omega$ with

$$
\mu_{1}\left(u_{4}, v_{4}\right)=0, \mu_{2}\left(u_{4}, v_{4}\right)<0
$$

Define, with $\tau_{1}>0$ and $\tau_{3}:=\tau_{1} \frac{\mu_{1}\left(u_{2}, v_{2}\right)-\mu_{2}\left(u_{2}, v_{2}\right)}{\mu_{2}\left(u_{4}, v_{4}\right)}>0$,

$$
\left(u^{-}(t), v^{-}(t)\right)=\left\{\begin{array}{ll}
\left(u_{2}, v_{2}\right) & \text { for } t \in\left[0, \tau_{1}\right] \\
\left(u_{4}, v_{4}\right) & \text { for } t \in\left(\tau_{1}, \tau_{3}+\tau_{1}\right]
\end{array} .\right.
$$

Then it follows that

$$
\begin{aligned}
\varphi\left(\tau_{3}+\tau_{1}, s^{-}, u^{-}, v^{-}\right) & =\varphi\left(\tau_{3}, \varphi\left(\tau_{1}, s^{-}, u_{2}, v_{2}\right), u_{4}, v_{4}\right) \\
& =\left[\begin{array}{cc}
e^{0} & 0 \\
0 & e^{\tau_{3} \mu_{2}\left(u_{4}, v_{4}\right)}
\end{array}\right]\left[\begin{array}{c}
e^{\tau_{1} \mu_{1}\left(u_{2}, v_{2}\right)} \\
e^{\tau_{1} \mu_{2}\left(u_{2}, v_{2}\right)}
\end{array}\right] s^{-}=e^{\tau_{1} \mu_{1}\left(u_{2}, v_{2}\right)} s^{-}
\end{aligned}
$$

Thus also the second equality in (3.2) holds with $\sigma^{-}=\tau_{3}+\tau_{1}$ and $\alpha^{-}=e^{\tau_{1} \mu_{1}\left(u_{2}, v_{2}\right)}<$ 1. Now Theorem 3.2 implies that there are four control set in $\mathbb{R}^{2}$ given by the interiors of the four quadrants.

Observe that conditions (3.4), (3.5), and (3.6) are satisfied in the simple example with $A(u, v)=\left[\begin{array}{ll}u & 0 \\ 0 & v\end{array}\right]$ and $\Omega=[-1,1] \times[-1,1]$. Then $\mu_{1}(u, v)=u, \mu_{2}(u, v)=v$, and one may choose

$$
\left(u_{1}, v_{1}\right)=(1,-1),\left(u_{2}, v_{2}\right)=(-1,1),\left(u_{3}, v_{3}\right)=(0,1),\left(u_{4}, v_{4}\right)=(0,-1) .
$$

The next example shows that the situation is quite different if $A$ is a two-dimensional Jordan block; in particular, scalar controls suffice to verify assumption (3.2) in Theorem 3.2 for a control set $\mathbb{S} D \neq \mathbb{S}^{1}$.

Example 3.8 Consider

$$
\left[\begin{array}{l}
\dot{x} \\
\dot{y}
\end{array}\right]=\left(\left[\begin{array}{ll}
\lambda & 1 \\
0 & \lambda
\end{array}\right]+u(t)\left[\begin{array}{cc}
b_{11} & b_{12} \\
0 & b_{11}
\end{array}\right]\right)\left[\begin{array}{l}
x \\
y
\end{array}\right],
$$

with $\lambda \in \mathbb{R}$ and $u(t) \in \Omega$. The system can be written as

$$
\left[\begin{array}{c}
\dot{x} \\
\dot{y}
\end{array}\right]=\left[\begin{array}{cc}
\lambda+b_{11} u & 1+b_{12} u \\
0 & \lambda+b_{11} u
\end{array}\right]\left[\begin{array}{l}
x \\
y
\end{array}\right]=A(u)\left[\begin{array}{l}
x \\
y
\end{array}\right] .
$$

For all $u \in \Omega$ the eigenvalue $\mu(u)=\lambda+b_{11} u$ has the eigenspace $\mathbb{R} \times\{0\}$. The intersection of the unit circle with the eigenspace is given by $\left\{(1,0)^{\top},(-1,0)^{\top}\right\}$, which are fixed under any control for the projected system. Suppose that $b_{12} \neq 0$ and 
$\Omega$ contains the two points $u_{1}:=0$ and $u_{2}:=-2 / b_{12}$, and write $\mu_{1}=\mu\left(u_{1}\right)=\lambda$ and $\mu_{2}=\mu\left(u_{2}\right)=\lambda-2 \frac{b_{11}}{b_{12}}$. Thus we consider the two differential equations

$$
\left[\begin{array}{c}
\dot{x} \\
\dot{y}
\end{array}\right]=\left[\begin{array}{cc}
\mu_{1} & 1 \\
0 & \mu_{1}
\end{array}\right]\left[\begin{array}{l}
x \\
y
\end{array}\right] \text { and }\left[\begin{array}{l}
\dot{x} \\
\dot{y}
\end{array}\right]=-\left[\begin{array}{cc}
-\mu_{2} & 1 \\
0 & -\mu_{2}
\end{array}\right]\left[\begin{array}{l}
x \\
y
\end{array}\right] .
$$

The solutions of (3.8) are given by

$$
\psi_{1}\left(t, x_{0}, y_{0}\right)=e^{\mu_{1} t}\left[\begin{array}{c}
x_{0}+t y_{0} \\
y_{0}
\end{array}\right], \quad \psi_{2}\left(t, x_{0}, y_{0}\right)=e^{\mu_{2} t}\left[\begin{array}{c}
x_{0}-t y_{0} \\
y_{0}
\end{array}\right],
$$

resp. For the projected systems on the unit circle the trajectory on the upper half-plane of the first equation tends for $t \rightarrow \infty$ to $(1,0)$ and for $t \rightarrow-\infty$ to $(-1,0)$. The trajectory for the second equation moves in the opposite direction. This proves that the open upper semicircle on $\mathbb{S}^{1}$ is an invariant control set ${ }_{\mathbb{S}} D_{1}$. Analogously, also the open lower semicircle on $\mathbb{S}^{1}$ is an invariant control set ${ }_{\mathbb{S}} D_{2}$.

In order to verify the conditions in (3.2) fix a point $s^{+} \in{ }_{\mathbb{S}} D_{1}$. Let $\tau>0$ and define

$$
u^{+}(t)=\left\{\begin{array}{ll}
u_{1} & \text { for } t \in[0, \tau] \\
u_{2} & \text { for } t \in(\tau, 2 \tau]
\end{array} .\right.
$$

It follows that

$$
\varphi\left(2 \tau, s^{+}, u^{+}\right)=\psi_{2}\left(\tau, \psi_{1}\left(\tau, s^{+}\right)\right)=e^{\mu_{2} \tau+\mu_{1} \tau} s^{+} .
$$

Then $\alpha^{+}=e^{\mu_{2} \tau+\mu_{1} \tau}>1$ if and only if $\mu_{2}+\mu_{1}=2 \lambda-2 \frac{b_{11}}{b_{12}}>0$, i.e., $\lambda>\frac{b_{11}}{b_{12}}$. Similarly, we can find conditions for $\alpha^{-}<1$ : The control sets on the unit sphere do not change if we add a third control value $u_{3}$ which will be specified in a moment. Repeating the derivation above, we find with $\mu_{3}:=\mu\left(u_{3}\right)$ that $\alpha^{-}:=e^{\mu_{3} \tau+\mu_{2} \tau}<1$ if and only if $\mu_{3}+\mu_{2}=\lambda+b_{11} u_{3}+\lambda-2 \frac{b_{11}}{b_{12}}<0$. This is equivalent to

$$
u_{3} b_{11}<2 \frac{b_{11}}{b_{12}}-2 \lambda
$$

We conclude that condition (3.2) holds if $\lambda>\frac{b_{11}}{b_{12}}$ for $\Omega=\left\{u_{1}, u_{2}, u_{3}\right\}$ with $u_{1}=0$ and $u_{2}=-\frac{2}{b_{12}}$, and $u_{3}$ satisfying (3.9). Then there are two invariant control sets with nonvoid interior in $\mathbb{R}^{2}$ given by the open upper and lower half-planes. Observe that these conditions hold, e.g., for

$$
\lambda=1, b_{11}=1, b_{12}=2 \text {, and } u_{1}=0, u_{2}=-1, u_{3}<-1 .
$$

Next we impose stronger assumptions on the homogeneous bilinear control system (2.7). We require that the control range $\Omega$ is a compact and convex neighborhood of the origin and that the accessibility rank condition holds on all of $\mathbb{P}^{n-1}$,

$$
\operatorname{dim} \mathcal{L} \mathcal{A}\{\mathbb{P} h(u, \cdot) ; u \in \Omega\}(p)=n-1 \text { for all } p \in \mathbb{P}^{n-1} .
$$


Then by Colonius and Kliemann [10, Theorem 7.1.1] there are $k_{0}$ control sets with nonvoid interior in $\mathbb{P}^{n-1}$ denoted by $\mathbb{P} D_{1}, \ldots, \mathbb{P} D_{k_{0}}, 1 \leq k_{0} \leq n$. Exactly one of these control sets is an invariant control set.

Remark 3.9 Braga Barros and San Martin [6] use the classification of semisimple Lie groups acting transitively on projective space $\mathbb{P}^{n-1}$ (cf. Boothby and Wilson [5]) to determine the number $k_{0} \in\{1, \ldots, n\}$ of control sets ${ }_{\mathbb{P}} D_{i}$ in projective space (it is either equal to $n, n / 2$, or $n / 4)$.

Next we analyze the relations between the control sets for the induced systems on projective space $\mathbb{P}^{n-1}$ and on the unit sphere $\mathbb{S}^{n-1}$. We will frequently use the following elementary facts that follow from (2.8):

Let $s_{1}, s_{2} \in \mathbb{S}^{n-1}$. If $s_{2}$ can be reached from $s_{1}$ (for system (3.1)), then $-s_{2}$ can be reached from $-s_{1}$. If on $\mathbb{P}^{n-1}$ the point $\mathbb{P}_{2}$ can be reached from $\mathbb{P} s_{1}$, then on $\mathbb{S}^{n-1}$ at least one of the points $s_{2}$ or $-s_{2}$ can be reached from $s_{1}$.

The proof of the following lemma is modeled after Bacciotti and Vivalda [2, Lemma 3], where controllable systems are analyzed.

Lemma 3.10 (i) Let $\mathbb{S}_{\mathbb{S}} D$ be a control set on $\mathbb{S}^{n-1}$. Then the projection of $\mathbb{S}_{\mathbb{S}} D$ to $\mathbb{P}^{n-1}$ is contained in a control set $\mathbb{P}_{\mathbb{P}} D$. (ii) Assume that the accessibility rank condition (3.10) on $\mathbb{P}^{n-1}$ holds and consider a control set $\mathbb{P}_{\mathbb{P}} D_{i}$ on $\mathbb{P}^{n-1}$. Suppose that there is $s_{0} \in \mathbb{S}^{n-1}$ such that $\mathbb{P}_{s_{0}} \in$ int $\left(\mathbb{P} D_{i}\right)$ and $-s_{0}$ can be reached from $s_{0}$. Then there exists a control set $\mathbb{S} D$ on $\mathbb{S}^{n-1}$ containing $A:=\left\{s \in \mathbb{S}^{n-1} \mid \mathbb{P} s \in \mathbb{P} D_{i}\right\}$.

Proof Assertion (i) is immediate from the definitions. Concerning assertion (ii) it is clear that for all $s \in A$ there is a control $u$ such that the trajectory of system (3.1) remains in $A$ for all $t \geq 0$. Now let $s_{1}, s_{2} \in A$. We have to show that $s_{2}$ is in the closure of the reachable set $\mathcal{O}^{+}\left(s_{1}\right)$ for system (3.1). Since $\mathbb{P}_{s_{1}}, \mathbb{P}_{s_{2}} \in \mathbb{P} D_{i}$ it follows that $s_{2} \in \overline{\mathcal{O}^{+}\left(s_{1}\right)}$ or $-s_{2} \in \overline{\mathcal{O}^{+}\left(s_{1}\right)}$. In the first case we are done. In the second case it follows that $s_{2} \in \overline{\mathcal{O}^{+}\left(-s_{1}\right)}$, and that, by our assumption, $s_{0} \in \mathcal{O}^{+}\left(-s_{0}\right)$. As noted in Sect. 2, $\mathbb{P}\left(-s_{1}\right)=\mathbb{P} s_{1} \in \mathbb{P} D_{i}$ and $\mathbb{P} s_{0} \in$ int $\left(\mathbb{P} D_{i}\right)$ imply that $\mathbb{P} s_{0}$ can be reached from $\mathbb{P}\left(-s_{1}\right)$, hence $s_{0} \in \mathcal{O}^{+}\left(-s_{1}\right)$ or $-s_{0} \in \mathcal{O}^{+}\left(-s_{1}\right)$. We claim that also in the second case one can reach $s_{0}$ from $-s_{1}$. In fact, $-s_{0} \in \mathcal{O}^{+}\left(-s_{1}\right)$ implies $s_{0} \in \mathcal{O}^{+}\left(-s_{0}\right) \subset \mathcal{O}^{+}\left(-s_{1}\right)$. Hence $s_{0} \in \mathcal{O}^{+}\left(-s_{1}\right)$ and we find $-s_{0} \in \mathcal{O}^{+}\left(s_{1}\right)$.

Since $\mathbb{P}_{s_{0}}, \mathbb{P}_{s_{2}} \in \mathbb{P} D$ it follows that $s_{2} \in \overline{\mathcal{O}^{+}\left(s_{0}\right)}$ or $-s_{2} \in \overline{\mathcal{O}^{+}\left(s_{0}\right)}$. In the second case, $s_{2} \in \overline{\mathcal{O}^{+}\left(-s_{0}\right)} \subset \overline{\mathcal{O}^{+}\left(s_{1}\right)}$ and in the first case, one has

$$
s_{2} \in \overline{\mathcal{O}^{+}\left(s_{0}\right)} \subset \overline{\mathcal{O}^{+}\left(-s_{0}\right)} \subset \overline{\mathcal{O}^{+}\left(s_{1}\right)}
$$

The proof of the next proposition uses arguments from Bacciotti and Vivalda [2, Proposition 2].

Proposition 3.11 If accessibility rank condition (3.10) holds for the induced system on $\mathbb{P}^{n-1}$, it also holds for the induced system on $\mathbb{S}^{n-1}$. 
Proof Recall that $\mathbb{P}^{n-1}=\left(\mathbb{R}^{n} \backslash\{0\}\right) / \sim$, where $\sim$ is the equivalence relation $x \sim y$ if $y=\lambda x$ with some $\lambda \neq 0$. Furthermore, an atlas of $\mathbb{P}^{n-1}$ is given by $n$ charts $\left(U_{i}, \psi_{i}\right)$, where $U_{i}$ is the set of equivalence classes $\left[x_{1}: \cdots: x_{n}\right]$ with $x_{i} \neq 0$ (the homogeneous coordinates) and $\psi_{i}: U_{i} \rightarrow \mathbb{R}^{n-1}$ is defined by

$$
\psi_{i}\left(\left[x_{1}: \cdots: x_{n}\right]\right)=\left(\frac{x_{1}}{x_{i}}, \ldots, \frac{\hat{x}_{i}}{x_{i}}, \ldots, \frac{x_{n}}{x_{i}}\right),
$$

where the hat means that the $i$-th entry is missing.

For the sake of simplicity we prove the rank condition for the North Pole of $\mathbb{S}^{n-1}$ given by $\bar{z}_{0}=(0, \ldots, 0,1)$. By assumption, the rank of the Lie algebra of the system on $\mathbb{P}^{n-1}$ is $n-1$ on all of $\mathbb{P}^{n-1}$. Consider the point $x_{0}=[0: \cdots: 0: 1] \in \mathbb{P}^{n-1}$. Thus there exist $n-1$ matrices $A_{1}, \ldots, A_{n-1}$ in the Lie algebra generated by the system on $\mathbb{R}^{n} \backslash\{0\}$ such that for the induced vector fields $A_{1}^{b}, \ldots, A_{n-1}^{b}$ in the Lie algebra for the system on $\mathbb{P}^{n-1}$ one obtains that the rank of the family $\left(A_{1}^{b}\left(x_{0}\right), \ldots, A_{n-1}^{b}\left(x_{0}\right)\right)$ is $n-1$. Now [2, formula (5)] shows the following formula for the local expression of this family, which has the form $\left(A_{1}^{n}\left(z_{0}\right), \ldots, A_{n-1}^{n}\left(z_{0}\right)\right)$ with $z_{0}=(0, \ldots, 0)$; let $a_{1}^{k}\left(\bar{z}_{0}\right), \ldots, a_{n}^{k}\left(\bar{z}_{0}\right)$ denote the $n$ components of $A_{k} \bar{z}_{0}$. Then, for $k=1, \ldots, n-1$,

$$
A_{k}^{n}\left(z_{0}\right)=\left(a_{1}^{k}\left(\bar{z}_{0}\right), \ldots, a_{n-1}^{k}\left(\bar{z}_{0}\right)\right)^{\top}-a_{n}^{k}\left(\bar{z}_{0}\right) z_{0}=\left(a_{1}^{k}\left(\bar{z}_{0}\right), \ldots, a_{n-1}^{k}\left(\bar{z}_{0}\right)\right)^{\top}
$$

So $A_{k}^{n}\left(z_{0}\right)$ is the vector whose components are equal to the first $n-1$ components of the last column of the matrix $A_{k}$.

On the other hand, the projections on $\mathbb{S}^{n-1}$ of the linear vector fields for the matrices $A_{1}, \ldots, A_{n-1}$ are the vector fields (cf. (3.1))

$$
A_{k}^{\circ}(x)=A_{k} x-x^{\top} A x \cdot x, \quad x \in \mathbb{S}^{n-1} .
$$

Thus we get, for $k=1, \ldots, n-1$

$$
A_{k}^{\circ}\left(\bar{z}_{0}\right)=A_{k} \bar{z}_{0}-\bar{z}_{0}^{\top} A_{k} \bar{z}_{0} \cdot \bar{z}_{0}=\left(a_{1}^{k}\left(\bar{z}_{0}\right), \ldots, a_{n-1}^{k}\left(\bar{z}_{0}\right), a_{n}^{k}\left(z_{0}\right)-\bar{z}_{0}^{\top} A_{k} \bar{z}_{0}\right)^{\top},
$$

so the $n-1$ first components of $A_{k}^{\circ}\left(\bar{z}_{0}\right)$ are equal to the components of $A_{k}^{n}\left(z_{0}\right)$. This implies that the vectors $A_{1}^{\circ}\left(\bar{z}_{0}\right), \ldots, A_{n-1}^{\circ}\left(\bar{z}_{0}\right)$ are linearly independent.

We get the following result characterizing the relation between the control sets ${ }_{\mathbb{P}} D_{1}, \ldots, \mathbb{P} D_{k_{0}}, 1 \leq k_{0} \leq n$, on projective space and the control sets on the unit sphere.

Theorem 3.12 Suppose that accessibility rank condition (3.10) holds for the induced system on projective space $\mathbb{P}^{n-1}$.

(i) If there is $s_{0} \in \mathbb{S}^{n-1}$ with $\mathbb{P} s_{0} \in$ int $\left(\mathbb{P}_{\mathbb{P}} D_{i}\right)$ such that $-s_{0}$ can be reached for system (3.1) from $s_{0}$, then $\mathbb{S}_{\mathbb{S}} D:=\left\{s \in \mathbb{S}^{n-1} \mid \mathbb{P}_{s} \in \mathbb{P} D_{i}\right\}$ is the unique control set on $\mathbb{S}^{n-1}$ which projects to $\mathbb{P} D_{i}$. 
(ii) For every control set ${ }_{\mathbb{P}} D_{i}, i \in\left\{1, \ldots, k_{0}\right\}$, there are at most two control sets $\mathbb{S}_{\mathbb{S}} D$ and $\mathbb{S}_{\mathbb{S}} D^{\prime}$ on $\mathbb{S}^{n-1}$ with nonvoid interior such that

$$
\left\{s \in \mathbb{S}^{n-1} \mid \mathbb{P}_{s} \in \mathbb{P}_{i} D_{i}={ }_{\mathbb{S}} D \cup{ }_{\mathbb{S}} D^{\prime},\right.
$$

and $\mathbb{S}_{\mathbb{S}}=-{ }_{\mathbb{S}} D^{\prime}$.

(iii) There are $k_{1}$ control sets with nonvoid interior on $\mathbb{S}^{n-1}$ denoted by ${ }_{\mathbb{S}} D_{1}, \ldots$, ${ }_{\mathbb{S}} D_{k_{1}}$ with $1 \leq k_{1} \leq 2 k_{0} \leq 2 n$. At most two of the sets ${ }_{\mathbb{S}} D_{i}$ are invariant control sets.

Proof (i) Suppose that there is $\mathbb{P} s_{0} \in$ int $\left(\mathbb{P} D_{i}\right)$ with $-s_{0} \in \mathcal{O}^{+}\left(s_{0}\right)$. By Lemma 3.10(ii) there is a control set ${ }_{S} D$ on the unit sphere containing $\left\{s \in \mathbb{S}^{n-1} \mid \mathbb{P}_{s} \in \mathbb{P} D_{i}\right\}$, hence the projection of ${ }_{\mathbb{S}} D$ to projective space contains $\mathbb{P}_{\mathbb{P}} D_{i}$. Using Lemma 3.10(i) one concludes that ${ }_{\mathbb{S}} D=\left\{s \in \mathbb{S}^{n-1} \mid \mathbb{P}_{s} \in \mathbb{P} D_{i}\right\}$.

(ii) Fix a point $s_{0} \in \mathbb{S}^{n-1}$ with $\mathbb{P} s_{0} \in \operatorname{int}\left(\mathbb{P} D_{i}\right)$ and define

$$
\begin{aligned}
& A^{+}:=\left\{s \in \mathbb{S}^{n-1} \mid \mathbb{P} s \in \mathbb{P}_{i} \text { and } s \in \mathcal{O}^{+}\left(s_{0}\right) \cap \mathcal{O}^{-}\left(s_{0}\right)\right\}, \\
& A^{-}:=\left\{s \in \mathbb{S}^{n-1} \mid \mathbb{P}_{s \in \mathbb{P}} D_{i} \text { and }-s \in \mathcal{O}^{+}\left(s_{0}\right) \cap \mathcal{O}^{-}\left(s_{0}\right)\right\} .
\end{aligned}
$$

The set $A^{+}$is contained in a control set ${ }_{\mathbb{S}} D$ and the set $A^{-}$is contained in a control set ${ }_{\mathbb{S}} D^{\prime}$. Every point $s$ with $\mathbb{P} s \in$ int $\left(\mathbb{P} D_{i}\right)$ satisfies $s \in \mathcal{O}^{+}\left(s_{0}\right)$ or $-s \in \mathcal{O}^{+}\left(s_{0}\right)$ and it also satisfies $s \in \mathcal{O}^{-}\left(s_{0}\right)$ or $-s \in \mathcal{O}^{-}\left(s_{0}\right)$. If there is $s \in \mathcal{O}^{+}\left(s_{0}\right)$ with $-s \in \mathcal{O}^{-}\left(s_{0}\right)$ hence $s \in \mathcal{O}^{-}\left(-s_{0}\right)$, it follows $-s_{0} \in \mathcal{O}^{+}\left(s_{0}\right)$. Then by part (i) the assertion follows. The same arguments apply if there is $s$ with $-s \in \mathcal{O}^{+}\left(s_{0}\right)$ and $s \in \mathcal{O}^{-}\left(s_{0}\right)$. Hence we may assume that either $s \in \mathcal{O}^{+}\left(s_{0}\right) \cap \mathcal{O}^{-}\left(s_{0}\right)$ or $-s \in \mathcal{O}^{+}\left(s_{0}\right) \cap \mathcal{O}^{-}\left(s_{0}\right)$. This shows that

$$
\left\{s \in \mathbb{S}^{n-1} \mid \mathbb{P} s \in \operatorname{int}\left(\mathbb{P} D_{i}\right)\right\} \subset A^{+} \cup A^{-} \subset\left({ }_{\mathbb{S}} D\right) \cup\left({ }_{\mathbb{S}} D^{\prime}\right) .
$$

It follows that $\left\{s \in \mathbb{S}^{n-1} \mid \mathbb{P} s \in \mathbb{P} D_{i}\right\} \subset \overline{\mathbb{S} D} \cup \overline{\mathbb{S} D^{\prime}}$, since $\mathbb{P}$ is an open map and ${ }_{\mathbb{P}} D_{i} \subset \overline{\operatorname{int}\left(\mathbb{P} D_{i}\right)}$. By Lemma 3.10(i) the projections of ${ }_{\mathbb{S}} D$ and ${ }_{\mathbb{S}} D^{\prime}$ to $\mathbb{P}^{n-1}$ are contained in $\mathbb{P} D_{i}$, hence (3.11) follows. The same arguments with $-s_{0}$ instead of $s_{0}$ implies that ${ }_{\mathbb{S}} D=-{ }_{\mathbb{S}} D^{\prime}$. If $\mathbb{S}_{\mathbb{S}} D$ or ${ }_{\mathbb{S}} D^{\prime}$ is an invariant control set, then also ${ }_{\mathbb{P}} D_{i}$ is an invariant control set, hence there are at most two invariant control set on $\mathbb{S}^{n-1}$.

(iii) This is a consequence of assertion (ii).

Recall the following definitions from Colonius and Kliemann [10]. For a solution $\varphi(t, x, u), t \geq 0$, of (2.7) the Lyapunov exponent is

$$
\lambda(u, x)=\limsup _{t \rightarrow \infty} \frac{1}{t} \log \|\varphi(t, x, u)\| .
$$

Observe that the Lyapunov exponents are constant on lines through the origin.

Definition 3.13 For a control set $\mathbb{P}_{\mathbb{P}}$ in $\mathbb{P}^{n-1}$ the Floquet spectrum is given by

$$
\Sigma_{F l}(\mathbb{P} D)=\left\{\begin{array}{l|l}
\lambda(u, x) & \begin{array}{c}
\mathbb{P} x \in \text { int }(\mathbb{P} D) \text { and } u \text { is piecewise constant } \\
\tau \text {-periodic for some } \tau \geq 0 \text { with } \mathbb{P} \varphi(\tau, x, u)=\mathbb{P} x
\end{array}
\end{array}\right\},
$$


and for a control set ${ }_{\mathbb{S}} D$ in $\mathbb{S}^{n-1}$ the Floquet spectrum is given by

$$
\Sigma_{F l}(\mathbb{S} D)=\left\{\begin{array}{l|l}
\lambda(u, x) & \begin{array}{c}
x \in \operatorname{int}(\mathbb{S} D) \text { and } u \text { is piecewise constant } \\
\tau \text {-periodic for some } \tau \geq 0 \text { with } s(\tau, x, u)=x
\end{array}
\end{array}\right\} .
$$

In the $\tau$-periodic case considered here the Lyapunov exponents satisfy $\lambda(u, x)=$ $\frac{1}{\tau} \log \|\varphi(\tau, x, u)\|$ for $\|x\|=1$ and coincide with the Floquet exponents (cf. Teschl $[26, \S 3.6])$. We note the following result.

Proposition 3.14 If ${ }_{S} D$ is a control set with nonvoid interior on $\mathbb{S}^{n-1}$ that projects to a control set $\mathbb{P} D$ in $\mathbb{P}^{n-1}$, then

$$
\Sigma_{F l}(\mathbb{S} D)=\Sigma_{F l}(\mathbb{P} D) .
$$

Proof The inclusion " $\Sigma_{F l}\left(\mathbb{S}_{\mathbb{S}} D\right) \subset \Sigma_{F l}(\mathbb{P} D)$ " is clear. For the converse, consider $\mathbb{P} x \in$ int $(\mathbb{P} D)$ and a piecewise constant $\tau$-periodic control $u$ with $\mathbb{P} \varphi(\tau, x, u)=\mathbb{P} x$. We may suppose that $x \in \mathbb{S}^{n-1}$, hence $x \in{ }_{\mathbb{S}} D$ or $-x \in{ }_{\mathbb{S}} D$. Consider the first case. If $\varphi(\tau, x, u)=\alpha x$ with $\alpha>0$ it follows that $\lambda(u, x)=\frac{1}{\tau} \log \alpha \in \Sigma_{F l}\left(\mathbb{S}_{\mathbb{S}} D\right)$. Otherwise $\varphi(\tau, x, u)=-\alpha x$ with $\alpha>0$ and hence

$$
\varphi(2 \tau, x, u)=\varphi(\tau, \varphi(\tau, x, u), u(\tau+\cdot))=-\alpha(-\alpha x)=\alpha^{2} x,
$$

implying

$$
\lambda(u, x)=\frac{1}{2 \tau} \log \|\varphi(2 \tau, x, u)\|=\frac{1}{2 \tau} \log \alpha^{2}=\frac{1}{\tau} \log \alpha \in \Sigma_{F l}\left(\mathbb{S}_{\mathbb{S}} D\right) .
$$

Analogously one argues in the case $-x \in{ }_{\mathbb{S}} D$.

The following result describes the control sets in $\mathbb{R}^{n}$ under the accessibility rank condition on projective space.

Theorem 3.15 Assume that the homogeneous bilinear control system (2.7) satisfies the accessibility rank condition (3.10) on $\mathbb{P}^{n-1}$. If a control set ${ }_{\mathbb{S}} D_{i}, i \in\left\{1, \ldots, k_{1}\right\}$, on $\mathbb{S}^{n-1}$ satisfies $0 \in \operatorname{int}\left(\Sigma_{F l}\left(\mathbb{S} D_{i}\right)\right)$, then the cone

$$
D_{i}=\left\{\alpha x \in \mathbb{R}^{n} \mid \alpha>0 \text { and } x \in{ }_{\mathbb{S}} D_{i}\right\}
$$

generated by ${ }_{\mathbb{S}} D_{i}$ is a control set with nonvoid interior in $\mathbb{R}^{n} \backslash\{0\}$. At most two of the $D_{i}$ are invariant control sets.

Proof By Proposition 3.11, every point in ${ }_{\mathbb{S}} D_{i}$ is locally accessible. Hence the first assertion follows from Theorem 3.2, if we can show that assumption (ii) in that theorem holds. The Floquet spectrum over a control set in projective space is a bounded interval, cf. [10, Proposition 6.2.14]. By Proposition 3.14 the same holds true for the Floquet spectrum of $\Sigma_{F l}\left(\mathbb{S} D_{i}\right)$. If $0 \in \operatorname{int}\left(\Sigma_{F l}\left(\mathbb{S} D_{i}\right)\right)$, it follows that there are points $s^{+}, s^{-} \in$ int $\left({ }_{\mathbb{S}} D_{i}\right)$, controls $u^{+}, u^{-} \in \mathcal{U}$ and times $\sigma^{+}, \sigma^{-}>0$ such that

$$
\varphi\left(\sigma^{+}, s^{+}, u^{+}\right)=\alpha^{+} s^{+}, \quad \varphi\left(\sigma^{-}, s^{-}, u^{-}\right)=\alpha^{-} s^{-}
$$


where $\alpha^{+}:=\exp \left(\sigma^{+} \lambda\left(u^{+}, s^{+}\right)\right) \in(1, \infty)$ and $\alpha^{-}:=\exp \left(\sigma^{-} \lambda\left(u^{-}, s^{-}\right)\right) \in(0,1)$. This verifies assumption (ii) of Theorem 3.2 if we take into account that we may vary $\sigma^{+}$and hence $\alpha^{+}$. Furthermore, every invariant control set $D$ projects to an invariant control set on $\mathbb{S}^{n-1}$, and here there are at most two invariant control sets.

Remark 3.16 Theorem 3.15 corrects Colonius and Kliemann [10, Corollary 12.2.6], [9, Theorem 7], where, assuming the stronger accessibility rank condition in $\mathbb{R}^{n} \backslash\{0\}$, a similar statement was given However, it was not taken into account that there may exist two control sets on the unit sphere that project to the same control set on projective space. It remains an open question if there are control sets $\mathbb{S}_{i} D_{i}$ with $0 \notin \operatorname{int}\left(\Sigma_{F l}\left(\mathbb{S} D_{i}\right)\right)$ that generate cones which are control sets on $\mathbb{R}^{n} \backslash\{0\}$.

Remark 3.17 Suppose that under the assumptions of Theorem 3.15 an invariant control set $D_{i}$ in $\mathbb{R}^{n} \backslash\{0\}$ exists. Then $D_{i} \cup\{0\}$ is a closed cone in $\mathbb{R}^{n}$ generated by an invariant control set on the unit sphere. If the system is not controllable, this cone does not coincide with $\mathbb{R}^{n}$, hence it is a nontrivial proper closed positively invariant cone in $\mathbb{R}^{n}$. On the other hand, Do Rocio et al. [11, Section 6] present an example in $\mathbb{R}^{4}$, which is not controllable and which also does not possess a nontrivial proper closed convex cone $W$ in $\mathbb{R}^{n}$ which is positively invariant. Here the convexity of $W$ is crucial: Such cones are pointed, i.e., $W \cap(-W)=\{0\}$, cf. [11, Lemma 4.1]. For an invariant control set $D_{i}$ as in Theorem 3.15 the cone $D_{i} \cup\{0\}$ need not be pointed (and hence not convex), since the invariant control set may contain the real eigenspace for a complex conjugate pair of eigenvalues of $A(u)$. Observe that here the convex closure of this cone, which is also positively invariant, coincides with $\mathbb{R}^{n}$. An example is the three-dimensional linear oscillator in Colonius and Kliemann [10, Example 10.2.3]. The existence of nontrivial proper closed convex positively invariant cones in $\mathbb{R}^{n}$ is analyzed in [11, Theorem 4.2, Theorem 4.5].

Not all control sets on the unit sphere generate cones that are control sets in $\mathbb{R}^{n} \backslash\{0\}$ as indicated by the following proposition,

Proposition 3.18 Assume that the homogeneous bilinear control system (2.7) satisfies the accessibility rank condition (3.10) on $\mathbb{S}^{n-1}$ and let $\mathbb{S} D$ be a control set in $\mathbb{S}^{n-1}$ with nonvoid interior. Then the following assertion holds.

If the supremum of $\{\lambda(u, x) \mid s(t, x, u) \in \mathbb{S} D$ for all $t \geq 0\}$ is less than 0 or the infimum is greater than 0 , then the cone

$$
C=\left\{\alpha x \in \mathbb{R}^{n} \mid \alpha>0 \text { and } x \in \mathbb{S} D\right\}
$$

is not a control set.

Proof Exact controllability to points in the interior of $\mathbb{S}_{\mathbb{S}} D$ implies that for all $x, y \in$ $\mathbb{R}^{n} \backslash\{0\}$ with $\frac{x}{\|x\|} \in{ }_{\mathbb{S}} D$ and $\frac{y}{\|y\|} \in \operatorname{int}\left({ }_{\mathbb{S}} D\right)$ there are $\alpha, T>0$ and $u \in \mathcal{U}$ with $\varphi(T, x, u)=\alpha y$. Now consider $(x, u)$ with $s(t, x, u) \in \mathbb{S} D$ for all $t \geq 0$. Then in the first case the trajectory in $\mathbb{R}^{n}$ satisfies $\| \varphi(t, x, u \| \rightarrow 0$ and in the second case it satisfies $\| \varphi(t, x, u \| \rightarrow \infty$. Hence the assertion follows. 
Remark 3.19 We refer to Colonius and Kliemann [10] for a discussion when the supremum and the infimum of $\left\{\lambda(u, x) \mid s(t, x, u) \in{ }_{\mathbb{S}} D\right.$ for all $\left.t \geq 0\right\}$ coincide with the supremum and the infimum of $\Sigma_{F l}(\mathbb{S} D)$, respectively. For dimension $n=2$, [10, Theorem 10.1.1] shows that these equalities hold if the accessibility rank condition holds in $\mathbb{P}^{1}$. For general $n \in \mathbb{N}$ suppose that the control range is given by $\rho \cdot \Omega, \rho \geq 0$, and the following " $\rho$-inner-pair condition" for the system on $\mathbb{S}^{n-1}$ holds:

For all $\rho^{\prime}>\rho$ every $(u, x) \in \mathcal{U} \times \mathbb{S}^{n-1}$ there is $t>0$ with $s(t, x, u) \in \operatorname{int}\left(\mathcal{O}^{+}(x)\right)$.

Then [10, Theorem 7.3.26] implies that for all $\rho \in(0, \infty)$ except for at most $n-1$ $\rho$-values the systems with control range $\rho \cdot \Omega$ have the property that the equalities for the suprema and the infima hold for all control sets.

The following example illustrates Theorem 3.15, cf. also [10, Examples 10.1.7 and 10.2.1] where for linear oscillators the spectral properties and the control sets in projective space are determined.

Example 3.20 Consider the damped linear oscillator

$$
\ddot{x}+3 \dot{x}+(1+u(t)) x=0 \text { with } u(t) \in \Omega=[-\rho, \rho],
$$

where $\rho \in\left(1, \frac{5}{4}\right)$. Hence the system equation is given by

$$
\left[\begin{array}{l}
\dot{x} \\
\dot{y}
\end{array}\right]=\left(\left[\begin{array}{cc}
0 & 1 \\
-1 & -3
\end{array}\right]+u\left[\begin{array}{cc}
0 & 0 \\
-1 & 0
\end{array}\right]\right)\left[\begin{array}{l}
x \\
y
\end{array}\right]=\left[\begin{array}{cc}
0 & 1 \\
-1-u & -3
\end{array}\right]\left[\begin{array}{l}
x \\
y
\end{array}\right] .
$$

The eigenvalues of $A(u)$ satisfy

$$
\operatorname{det}(\lambda I-A(u))=\operatorname{det}\left(\begin{array}{cc}
\lambda & -1 \\
1+u & \lambda+3
\end{array}\right)=\lambda^{2}+3 \lambda+1+u=0
$$

and one obtains two real eigenvalues

$$
\lambda_{1}(u)=-\frac{3}{2}-\sqrt{\frac{5}{4}-u} \text { and } \lambda_{2}(u)=-\frac{3}{2}+\sqrt{\frac{5}{4}-u}
$$

with corresponding eigenvectors $\left(x, \lambda_{1}(u) x\right)^{\top}$ and $\left(x, \lambda_{2}(u) x\right)^{\top}, x \neq 0$. Note that $\lambda_{2}(u)>0$ if and only if $u \in[-\rho,-1)$. Since for all $u \in[-\rho, \rho]$ one has $\lambda_{1}(u)<$ $\lambda_{2}(u)$ the projected trajectories in $\mathbb{P}^{1}$ go from the eigenspace for $\lambda_{1}(u)$ to the eigenspace for $\lambda_{2}(u)$. A short computation shows that there is an open control set $D_{\mathbb{P}}$ and a closed invariant control set ${ }_{\mathbb{P}} D_{2}$ in projective space $\mathbb{P}^{1}$ given by the projections of

$$
\left\{\left[\begin{array}{c}
x \\
\lambda x
\end{array}\right] \mid x \neq 0, \lambda \in \Sigma_{F l}\left(\mathbb{P} D_{1}\right)\right\}, \quad\left\{\left[\begin{array}{c}
x \\
\lambda x
\end{array}\right] \mid x \neq 0, \lambda \in \overline{\Sigma_{F l}\left(\mathbb{P} D_{2}\right)}\right\},
$$


resp., where by [10, Theorem 10.1.1] the Floquet spectra are

$$
\begin{aligned}
& \left.\Sigma_{F l(\mathbb{P}} D_{1}\right)=\left(-\frac{3}{2}-\sqrt{\frac{5}{4}+\rho},-\frac{3}{2}-\sqrt{\frac{5}{4}-\rho}\right) \subset(-\infty, 0), \\
& \Sigma_{F l}\left(\mathbb{P} D_{2}\right)=\left(-\frac{3}{2}+\sqrt{\frac{5}{4}-\rho},-\frac{3}{2}+\sqrt{\frac{5}{4}+\rho}\right) .
\end{aligned}
$$

The control sets in $\mathbb{P}^{1}$ induce four control sets on the unit circle $\mathbb{S}^{1}$. For $\mathbb{P} D_{2}$ one obtains the two control sets $\mathbb{S}_{\mathbb{S}} D_{2}^{\prime}=-{ }_{\mathbb{S}} D_{2}$. Since $u=-1 \in(-\rho, \rho)$ and $0=$ $\lambda_{2}(-1) \in$ int $\left(\Sigma_{F l}\left(\mathbb{P} D_{2}\right)\right)$, Theorem 3.15 implies that there are two invariant control sets in $\mathbb{R}^{2} \backslash\{0\}$, they are the cones

$$
D_{2}=\left\{\alpha\left[\begin{array}{l}
x \\
y
\end{array}\right] \mid \alpha>0,\left[\begin{array}{l}
x \\
y
\end{array}\right] \in{ }_{\mathbb{S}} D_{2}\right\}, D_{2}^{\prime}=\left\{\alpha\left[\begin{array}{l}
x \\
y
\end{array}\right] \mid \alpha>0,\left[\begin{array}{l}
x \\
y
\end{array}\right] \in{ }_{\mathbb{S}} D_{2}^{\prime}\right\} .
$$

Next we present a necessary and sufficient condition for controllability on $\mathbb{R}^{n} \backslash\{0\}$. The infimal and supremal Lyapunov exponents, cf. (3.12), are

$$
\kappa^{*}=\inf _{u \in \mathcal{U}} \inf _{x \neq 0} \lambda(u, x) \text { and } \kappa=\sup _{u \in \mathcal{U}} \sup _{x \neq 0} \lambda(u, x)
$$

resp. The following result improves Colonius and Kliemann [10, Corollary 12.2.6(iii)], where the accessibility rank condition is assumed in $\mathbb{R}^{n} \backslash\{0\}$.

Corollary 3.21 Assume that the homogeneous bilinear control system (2.7) satisfies the accessibility rank condition (3.10) on $\mathbb{P}^{n-1}$. Then it is controllable in $\mathbb{R}^{n} \backslash\{0\}$ if and only if the induced system on $\mathbb{P}^{n-1}$ is controllable and $\kappa^{*}<0<\kappa$.

Proof Controllability on $\mathbb{R}^{n} \backslash\{0\}$ implies controllability on $\mathbb{P}^{n-1}$. Furthermore, asymptotic null controllability to $0 \in \mathbb{R}^{n}$, and hence exponential null controllability follows by [10, Corollary 12.2.3]. Thus $\kappa^{*}<0$ and, by time reversal, also $\kappa>0$ follows.

Conversely, controllability on $\mathbb{P}^{n-1}$ implies by Bacciotti and Vivalda [2, Theorem 1] that ${ }_{\mathbb{S}} D=\mathbb{S}^{n-1}$ is a control set. By Theorem 3.15 , it follows that $\mathbb{R}^{n} \backslash\{0\}$ is a control set. This implies that for every initial point $x \neq 0$ the reachable set $\mathcal{O}^{+}(x)$ is dense in $\mathbb{R}^{n} \backslash\{0\}$, i.e., approximate controllability holds. For homogeneous bilinear control systems, Cannarsa and Sigalotti [7, Theorem 1] shows that approximate controllability implies controllability in $\mathbb{R}^{n} \backslash\{0\}$. This completes the proof.

Remark 3.22 The condition $\kappa^{*}<0<\kappa$ can be replaced by the requirement that $0 \in \operatorname{int}\left(\Sigma_{F l}\left(\mathbb{P}^{n-1}\right)\right)=\left(\kappa^{*}, \kappa\right)$. This follows, since by [10, Theorem 7.1.5(iv)] the Floquet spectrum is an interval and satisfies $\overline{\Sigma_{F l}\left(\mathbb{P}^{n-1}\right)}=\left[\kappa^{*}, \kappa\right]$ if $\mathbb{P}^{n-1}$ is a control set.

Remark 3.23 For control systems on semisimple Lie groups, San Martin [23, Proposition 5.6] shows the following result. Let $G \subset S l(n, \mathbb{R})$ be a semisimple, connected, and noncompact group acting transitively on $\mathbb{R}^{n} \backslash\{0\}$ and let $S$ be a semigroup with 
nonvoid interior in $G$. Then $S$ is controllable on $\mathbb{R}^{n} \backslash\{0\}$ if and only if $S$ is controllable in $\mathbb{P}^{n-1}$. In this case $0 \in\left(\kappa^{*}, \kappa\right)=\operatorname{int}\left(\Sigma_{F l}\left(\mathbb{P}^{n-1}\right)\right)$.

\section{Equilibria of affine systems}

In the rest of this paper we discuss control sets for affine systems of the form (1.1). We begin by analyzing the equilibria.

For each control value $u \in \Omega$, an associated equilibrium point of system (1.1) is a state $x_{u}$ that satisfies

$$
0=A(u) x_{u}+C u+d
$$

If for $u \in \Omega$ there is a solution $x_{u}$ of (4.1) and $\operatorname{det} A(u)=0$, then every point in the nontrivial affine subspace $x_{u}+\operatorname{ker} A(u)$ is an equilibrium. If there is $u \in \Omega$ with $C u+d=0$, then equation (4.1) always has the solution $x_{u}=0$. If $\operatorname{det} A(u) \neq 0$, then there exists a unique equilibrium of (1.1) given by

$$
x_{u}=-A(u)^{-1}[C u+d] .
$$

The following simple but useful result shows that for constant control $u$ the phase portrait of the inhomogeneous equation is obtained by shifting the origin to $x_{u}$.

Proposition 4.1 Consider for constant control $u \in \Omega$ a solution $\varphi(t, x, u), t \geq 0$, of the inhomogeneous equation (1.1) and let $x_{u}$ be an associated equilibrium. Then $\varphi(t, x, u)-x_{u}$ is a solution of the homogeneous equation $\dot{x}(t)=A(u) x(t)$ with initial value $x-x_{u}$.

Proof We compute

$$
\begin{aligned}
\frac{d}{d t}\left[\varphi(t, x, u)-x_{u}\right] & =A(u)\left[\varphi(t, x, u)-x_{u}\right]+A(u) x_{u}+C u+d \\
& =A(u)\left[\varphi(t, x, u)-x_{u}\right] .
\end{aligned}
$$

The following proposition shows that the affine control system (1.1) is equivalent to an inhomogeneous bilinear system, if there is $u^{0} \in \Omega$ with $C u^{0}+d=0$.

Proposition 4.2 Suppose that there is $u^{0} \in \Omega$ with $C u^{0}+d=0$ and consider

$$
\dot{x}(t)=A\left(u^{0}\right) x(t)+\sum_{i=1}^{m} v_{i}(t) B_{i} x(t)+C v(t) \text { with } v(t) \in \Omega^{\prime}:=\Omega-u^{0},
$$

with trajectories denoted by $\psi(\cdot, x, v)$. Then the trajectories $\varphi(\cdot, x, u), u \in \mathcal{U}$, of $(1.1)$ satisfy $\varphi(t, x, u)=\psi(t, x, v), t \in \mathbb{R}$, with controls $v(t)=u(t)-u^{0}, t \in \mathbb{R}$.

Proof One computes for a solution $x(t)=\varphi(t, x, u), t \in \mathbb{R}$, of (1.1) 


$$
\begin{aligned}
\dot{x}(t) & =A x(t)+\sum_{i=1}^{m} u_{i}^{0} B_{i} x(t)+\sum_{i=1}^{m}\left(u_{i}(t)-u_{i}^{0}\right) B_{i} x(t)+C\left(u(t)-u^{0}\right)+C u^{0}+d \\
& =A\left(u^{0}\right) x(t)+\sum_{i=1}^{m} v_{i}(t) B_{i} x(t)+C v(t) .
\end{aligned}
$$

We introduce the following notation for the set of equilibria,

$$
\begin{aligned}
E & =\left\{x \in \mathbb{R}^{n} \mid 0=A(u) x+C u+d \text { for some } u \in \Omega\right\}, \\
E_{0} & =\left\{x \in \mathbb{R}^{n} \mid 0=A(u) x+C u+d \text { for some } u \in \operatorname{int}(\Omega)\right\} .
\end{aligned}
$$

Note that $\overline{E_{0}}=E$ if $\Omega=\overline{\operatorname{int}(\Omega)}$. The following discussion of systems with scalar controls follows essentially Mohler [20, Section 2.4].

Theorem 4.3 Consider system (1.1) with scalar control and assume that for all $u \in \Omega$ it follows from $\operatorname{det}(A+u B)=0$ that there is no solution to Eq. (4.1). (i) Suppose that there is $u^{0} \in \Omega=\mathbb{R}$ with $A+u^{0} B$ nonsingular. Then there are at most $1 \leq r \leq n$ control values $v^{i} \in \mathbb{R}$ such that the equilibrium set is given by

$$
E=\left\{x_{u} \mid u \in \mathbb{R} \backslash\left\{v^{1}, \ldots, v^{r}\right\}\right\}
$$

and is the union of at most $n+1$ smooth curves. These curves have no finite endpoints. (ii) If $\Omega$ is a possibly unbounded interval, the equilibrium set $E$ has at most $n+1$ connected components.

Proof First note that $x_{u}=-(A+u B)^{-1}[C u+d]$ describes a smooth curve as long as $\operatorname{det}(A+u B) \neq 0$. Since $\operatorname{det}(A+u B)$ is a nontrivial polynomial in $u$ of degree at most $n$, there are most $n$ real roots $v^{1}, \ldots, v^{r}, 0 \leq r \leq n$, of $\operatorname{det}(A+u B)=0$. By our assumption the vectors $C v^{i}+d$ are not in the range of $A+v^{i} B$.

Consider a sequence $u^{k} \rightarrow v^{i}$ for some $i$. If $x_{u^{k}}$ remains bounded, we may assume that it converges to some $y \in \mathbb{R}^{n}$. For $k \rightarrow \infty$ we find

$$
\left(A+v^{i} B\right) y=-\left(C v^{i}+d\right)
$$

contradicting the assumption of the theorem. It follows that $x_{u^{k}}$ becomes unbounded for $k \rightarrow \infty$.

(ii) If $\Omega=\left[u_{*}, u^{*}\right], u_{*}<u^{*}$, the equilibrium set $E=\left\{x_{u} \mid u \in \Omega \backslash\left\{v^{1}, \ldots, v^{r}\right\}\right\}$ consists of at most $n+1$ smooth curves having no finite endpoints, with the possible exception of the equilibria corresponding to the minimum and maximum values of $u$ in $\Omega$, i.e., $u=u_{*}, u^{*}$. If there is more than one curve constituting $E$, then the finite end points which are the equilibria $x_{u_{*}}$ and $x_{u^{*}}$ must lie on different curves. Hence the assertion also follows in this case. Similarly, the assertion follows for intervals which are unbounded to one side. 
The following example is used in Rink and Mohler [21, Example 2] and Mohler [20, Example 2 on page 32] as an example for a system that is not controllable. It illustrates the result above.

Example 4.4 Consider the control system given by

$$
\left[\begin{array}{c}
\dot{x} \\
\dot{y}
\end{array}\right]=\left[\begin{array}{c}
2 u(t) x+y \\
x+2 u(t) y+u(t)
\end{array}\right]
$$

With

$$
A=\left[\begin{array}{ll}
0 & 1 \\
1 & 0
\end{array}\right], \quad B=\left[\begin{array}{ll}
2 & 0 \\
0 & 2
\end{array}\right], \quad C=\left[\begin{array}{l}
0 \\
1
\end{array}\right]
$$

this is the inhomogeneous bilinear control system

$$
\left[\begin{array}{c}
\dot{x} \\
\dot{y}
\end{array}\right]=\left[\begin{array}{cc}
2 u & 1 \\
1 & 2 u
\end{array}\right]\left[\begin{array}{l}
x \\
y
\end{array}\right]+\left[\begin{array}{l}
0 \\
1
\end{array}\right] u=(A+u B)\left[\begin{array}{l}
x \\
y
\end{array}\right]+C u .
$$

The eigenvalues of $A(u)=A+u B$ are given by $0=\operatorname{det}(A+u B)=4 u^{2}-1$, hence $\lambda_{1}(u)=2 u+1>\lambda_{2}(u)=2 u-1$. One finds $\lambda_{2}(u)>0$ for $u>\frac{1}{2}$ and $\lambda_{1}(u)<0$ for $u<-\frac{1}{2}$. For $u \in\left(-\frac{1}{2}, \frac{1}{2}\right)$ one gets $\lambda_{1}(u)>0$ and $\lambda_{2}(u)<0$, hence the matrix $A+u B$ is hyperbolic here.

For every $u \in \mathbb{R}$, the eigenspace for $\lambda_{1}(u)$ is $\operatorname{Diag}_{1}:=\left\{(z, z)^{\top} \mid z \in \mathbb{R}\right\}$ and the eigenspace for $\lambda_{2}(u)$ is $\operatorname{Diag}_{2}:=\left\{(z,-z)^{\top} \mid z \in \mathbb{R}\right\}$. For $|u| \neq \frac{1}{2}$ the equilibria are given by

$$
\left[\begin{array}{l}
x_{u} \\
y_{u}
\end{array}\right]=-(A+u B)^{-1} C u=\frac{-1}{4 u^{2}-1}\left[\begin{array}{cc}
2 u & -1 \\
-1 & 2 u
\end{array}\right]\left[\begin{array}{l}
0 \\
1
\end{array}\right] u=\frac{u}{4 u^{2}-1}\left[\begin{array}{c}
1 \\
-2 u
\end{array}\right] .
$$

Thus we see that

$$
y_{u}=-2 u x_{u} \text { for }|u| \neq \frac{1}{2}
$$

The assumption of Theorem 4.3 is satisfied, since for $u= \pm \frac{1}{2}$ there is no solution to

$$
\left[\begin{array}{l}
0 \\
0
\end{array}\right]=(A+u B)\left[\begin{array}{l}
x \\
y
\end{array}\right]+C u=\left[\begin{array}{cc} 
\pm 1 & 1 \\
1 & \pm 1
\end{array}\right]\left[\begin{array}{l}
x \\
y
\end{array}\right]+\left[\begin{array}{l}
0 \\
1
\end{array}\right]\left( \pm \frac{1}{2}\right)
$$

For the asymptotics of the equilibria, Eq. (4.5) shows that $\left(x_{u}, y_{u}\right)^{\top}$ approach the line $\operatorname{Diag}_{2}$ for $u \rightarrow \frac{1}{2}$ and the line $\operatorname{Diag}_{1}$ for $u \rightarrow-\frac{1}{2}$. In both cases, the equilibria become unbounded. For $u \rightarrow \pm \infty$, one obtains that the equilibria approach $\left(0,-\frac{1}{2}\right)^{\top}$. 
This discussion shows that the set of equilibria for unbounded control $u$ consists of the following three connected branches

$$
\begin{aligned}
& \mathcal{B}_{1}=\left\{\left[\begin{array}{l}
x_{u} \\
y_{u}
\end{array}\right] \mid u \in\left(-\frac{1}{2}, \frac{1}{2}\right)\right\}, \quad \mathcal{B}_{2}=\left\{\left[\begin{array}{l}
x_{u} \\
y_{u}
\end{array}\right] \mid u \in\left(-\infty,-\frac{1}{2}\right)\right\}, \\
& \mathcal{B}_{3}=\left\{\left[\begin{array}{l}
x_{u} \\
y_{u}
\end{array}\right] \mid u \in\left(\frac{1}{2}, \infty\right)\right\} .
\end{aligned}
$$

The equilibria in $\mathcal{B}_{2}$ and $\mathcal{B}_{3}$ both approach $\left(0,-\frac{1}{2}\right)^{\top}$ for $|u| \rightarrow \infty$; cf. also Mohler [20, Figure 2.1 on p. 33] or Rink and Mohler [21, Figure 1]. The equilibria in $\mathcal{B}_{2}$ are stable, those in $\mathcal{B}_{3}$ are totally unstable, and those in $\mathcal{B}_{1}$ yield one positive and one negative eigenvalue.

\section{Control sets and equilibria of affine systems}

The controllability properties near equilibria will be analyzed assuming that the linearized control systems are controllable. This yields results on the control sets around equilibria.

In order to describe the properties of the system linearized about an equilibrium, we recall the following classical result from Lee and Markus [19, Theorem 1 on p. 366].

Theorem 5.1 Consider the control process in $\mathbb{R}^{n}$

$$
\dot{x}=f(x, u),
$$

where $f$ is $C^{1}$ and suppose that $f(0,0)=0$ where 0 is in the interior of the control range $\Omega$. Then the controllable set $\mathcal{O}^{-}(0)$ is open if, with $A=\frac{\partial f}{\partial x}(0,0)$ and $B=$ $\frac{\partial f}{\partial u}(0,0)$,

$$
\operatorname{rank}\left[B, A B, \ldots, A^{n-1} B\right]=n
$$

Condition (5.2) is the familiar Kalman condition for controllability of the linearized system $\dot{x}=\frac{\partial f}{\partial x}(0,0) x+\frac{\partial f}{\partial u}(0,0) u$ (without control restriction), cf. Sontag [24, Theorem 3, p. 89].

We apply this result to affine control systems and obtain that the reachable set and the controllable set for an equilibrium are open, if the linearized system is controllable.

Proposition 5.2 Consider the affine system (1.1) and let $x_{u}$ be an equilibrium for a control value $u \in$ int $(\Omega)$, where the rank condition

$$
\operatorname{rank}\left[B^{\prime}(u), A(u) B^{\prime}(u), \ldots,(A(u))^{n-1} B^{\prime}(u)\right]=n
$$

holds with $B^{\prime}(u)$ defined by

$$
B^{\prime}(u)=C+\left[B_{1} x_{u}, \ldots, B_{m} x_{u}\right] .
$$


Then the reachable set $\mathcal{O}^{+}\left(x_{u}\right)$ and the controllable set $\mathcal{O}^{-}\left(x_{u}\right)$ are open. If $A(u)=$ $A+\sum_{i=1}^{m} u_{i} B_{i}$ is invertible, then

$$
B^{\prime}(u)=C-\left[B_{1} A(u)^{-1}(C u+d), \ldots, B_{m} A(u)^{-1}(C u+d)\right] .
$$

Proof First we convince ourselves that Theorem 5.1 can be applied to arbitrary equilibria $\left(x^{0}, u^{0}\right)$ with $u^{0} \in$ int $(\Omega)$ instead of $(0,0)$. In fact, define $\tilde{f}(x, u):=$ $f\left(x+x^{0}, u+u^{0}\right)$. Then $(0,0)$ is an equilibrium of

$$
\dot{x}(t)=\tilde{f}(x(t), u(t)) \text { with control range } \Omega-u^{0},
$$

and the control value $u=0$ is $\operatorname{in} \operatorname{int}\left(\Omega-u^{0}\right)$. The solutions $\psi(t, 0, u), t \geq 0$, of (5.5) are given by $\varphi\left(t, x^{0}, u+u^{0}\right)-x^{0}$, since $\varphi\left(0, x^{0}, u+u^{0}\right)-x^{0}=0$ and

$$
\begin{aligned}
\frac{d}{d t}\left[\varphi\left(t, x^{0}, u+u^{0}\right)-x^{0}\right] & =f\left(\varphi\left(t, x^{0}, u+u^{0}\right), u(t)+u^{0}\right) \\
& =\tilde{f}\left(\varphi\left(t, x^{0}, u+u^{0}\right)-x^{0}, u(t)\right) .
\end{aligned}
$$

Hence $\mathcal{O}^{-}\left(x^{0}\right)$ coincides with the controllable set $\tilde{\mathcal{O}}^{-}(0)$ of (5.5). The rank condition (5.2) for (5.5) involves

$$
A=\frac{\partial \tilde{f}}{\partial x}(0,0)=\frac{\partial f}{\partial x}\left(x^{0}, u^{0}\right), B=\frac{\partial \tilde{f}}{\partial u}(0,0)=\frac{\partial f}{\partial u}\left(x^{0}, u^{0}\right) .
$$

For system (1.1) $f(x, u)=A(u) x+C u+d$ and for an equilibrium $x_{u}$ we find $\frac{\partial f}{\partial x}\left(x_{u}, u\right)=A(u)$ and

$$
\frac{\partial f}{\partial u}\left(x_{u}, u\right)=C+\frac{\partial}{\partial u} \sum_{i=1}^{m} u_{i} B_{i} x_{u}=C+\left[B_{1} x_{u}, \ldots, B_{m} x_{u}\right]
$$

By (5.3) the rank condition (5.2) is satisfied. Applying Theorem 5.1 we conclude that the controllable set $\mathcal{O}^{-}\left(x_{u}\right)$ is open. By time reversal, cf. Lemma 2.2, also the reachable set $\mathcal{O}^{+}\left(x_{u}\right)$ is open.

If $A(u)$ is invertible, the formula for $B^{\prime}(u)$ follows from (4.2).

The following proposition shows that the controllability rank condition (5.3) holds generically for controls $u \in \mathbb{R}^{m}$ if it holds in some $u^{0}$.

Proposition 5.3 Assume that $A(u)$ is invertible for all $u \in \mathbb{R}^{m}$ and that the rank condition (5.3) holds for some $u^{0} \in \mathbb{R}^{m}$. Then (5.3) holds for all $u$ in an open and dense subset of $\mathbb{R}^{m}$.

Proof Define

$$
B^{\prime \prime}(u):=\operatorname{det} A(u) C-\left[B_{1} \operatorname{Adj}(A(u))(C u+d), \ldots, B_{m} \operatorname{Adj}(A(u))(C u+d)\right],
$$


where $\operatorname{Adj}(A(u))$ is defined by $(A(u))^{-1} \operatorname{det} A(u)=\operatorname{Adj}(A(u))$. Condition (5.3) holds if and only if

$$
\operatorname{rank}\left[B^{\prime \prime}(u), A(u) B^{\prime \prime}(u), \ldots,(A(u))^{n-1} B^{\prime \prime}(u)\right]=n .
$$

The entries of the matrix in (5.6) are polynomial in the variables $u_{1}, \ldots, u_{m}$. Using the assumption one finds that the set of $u \in \mathbb{R}^{m}$ violating (5.6) is contained in a proper algebraic variety; the complement of such a set is open and dense in $\mathbb{R}^{m}$ (this follows in the same way as the genericity of the controllability rank condition (5.2), cf. Sontag [24, Proposition 3.3.12]).

Remark 5.4 For a system of the form (1.1) with scalar control the assumptions of Proposition 5.3 imply that there are at most finitely many $u$ such that the rank condition (5.3) is violated. This follows taking into account that for scalar $u$ the entries of the matrix in (5.6) are polynomial in the scalar variable $u$, hence there are at most finitely many zeros.

A consequence of Proposition 5.2 is the following first result on control sets.

Proposition 5.5 Consider the affine system (1.1) and assume that the rank condition (5.3) is satisfied for some $u \in \operatorname{int}(\Omega)$. Then the set $D=\mathcal{O}^{-}\left(x_{u}\right) \cap \overline{\mathcal{O}^{+}\left(x_{u}\right)}$ is a control set of system (1.1) containing the equilibrium $x_{u}$ in the interior.

Proof By Proposition 5.2 the sets $\mathcal{O}^{-}\left(x_{u}\right)$ and $\mathcal{O}^{+}\left(x_{u}\right)$ are open neighborhoods of $x_{u}$, hence it follows that $x_{u}$ is in the interior of the set $D_{0}:=\mathcal{O}^{-}\left(x_{u}\right) \cap \overline{\mathcal{O}^{+}\left(x_{u}\right)}$.

Let $x \in D_{0}$. Then $x_{u} \in \mathcal{O}^{+}(x)$ and therefore $\mathcal{O}^{+}\left(x_{u}\right) \subset \mathcal{O}^{+}(x)$ and as $D_{0} \subset$ $\overline{\mathcal{O}^{+}\left(x_{u}\right)}$, it follows that $D_{0} \subset \overline{\mathcal{O}^{+}(x)}$. Next we show that there is a control $v \in \mathcal{U}$ with $\varphi(t, x, v) \in D_{0}$ for all $t \geq 0$. Since $x \in \mathcal{O}^{-}\left(x_{u}\right)$ there are $T>0$ and $v_{1} \in \mathcal{U}$ such that $\varphi\left(T, x, v_{1}\right)=x_{u}$ and $\varphi\left(t, x, v_{1}\right) \in \mathcal{O}^{-}\left(x_{u}\right)$ for all $t \in[0, T]$. Furthermore, $\varphi\left(t, x, v_{1}\right) \in \mathcal{O}^{+}(x)$ and $x \in \overline{\mathcal{O}^{+}\left(x_{u}\right)}$, and hence continuous dependence on the initial value shows that $\varphi\left(t, x, v_{1}\right) \in \overline{\mathcal{O}^{+}\left(x_{u}\right)}$ for all $t \in[0, T]$. Now the control function

$$
v(t):= \begin{cases}v_{1}(t) & \text { for } t \in[0, T] \\ u & \text { for } t>T\end{cases}
$$

yields $\varphi(t, x, v) \in D_{0}$ for all $t \geq 0$. We have shown that $D_{0}$ satisfies properties (i) and (ii) in Definition 2.1. Hence it is contained in a maximal set $D$ with these properties, i.e., a control set, obtained as the union of all sets satisfying properties (i) and (ii) and containing $D_{0}$.

Let us show that $D_{0}=D$. By the definition of control sets and $x_{u} \in D$, the inclusion $D \subset \overline{\mathcal{O}^{+}\left(x_{u}\right)}$ holds and for $x \in D$ one has $x_{u} \in \overline{\mathcal{O}^{+}(x)}$. Using that $\mathcal{O}^{-}\left(x_{u}\right)$ is a neighborhood of $x_{u}$ this implies that there are $T>0$ and a control $u \in \mathcal{U}$ with $\varphi(T, x, u) \in \mathcal{O}^{-}\left(x_{u}\right)$, and hence $x \in \mathcal{O}^{-}\left(x_{u}\right)$. This shows that $D \subset$ $\mathcal{O}^{-}\left(x_{u}\right) \cap \overline{\mathcal{O}^{+}\left(x_{u}\right)}=D_{0}$ and hence equality holds concluding the proof that $D_{0}$ is a control set.

Next we show that every connected subset of the set $E_{0}$ of equilibria is contained in a single control set, if the systems linearized about the equilibria are controllable. 
Theorem 5.6 Let $\mathcal{C} \subset\left\{x_{u} \mid u \in\right.$ int $\left.(\Omega)\right\}=E_{0}$ be a pathwise connected subset of the set of equilibria of system (1.1) and assume that for every equilibrium $x_{u}$ in $\mathcal{C}$ the control $u$ satisfies the rank condition (5.3). Then there exists a control set D containing $\mathcal{C}$ in the interior and $D=\mathcal{O}^{-}\left(x_{u}\right) \cap \overline{\mathcal{O}^{+}\left(x_{u}\right)}$ for every $x_{u} \in \mathcal{C}$.

Proof By Proposition 5.5 every equilibrium $x_{u} \in \mathcal{C}$ is contained in the interior of a control set. Consider two points $x_{u}$ and $x_{v}$ in $\mathcal{C}$. Then $x_{v} \in \mathcal{O}^{+}\left(x_{u}\right)$. In fact, consider a continuous path from $x_{u}$ to $x_{v}$ in $\mathcal{C}$, say $h:[0,1] \rightarrow \mathcal{C}$ with $h(0)=x_{u}$ and $h(1)=x_{v}$. Let

$$
\tau:=\sup \left\{s \in[0,1] \mid \forall s^{\prime} \in[0, s]: h\left(s^{\prime}\right) \in \mathcal{O}^{+}\left(x_{u}\right)\right\}
$$

Observe that $\tau>0$, since by Proposition 5.2, the reachable set $\mathcal{O}^{+}\left(x_{u}\right)$ is open. If $\tau<1$, then $y:=h(\tau) \in \overline{\mathcal{O}^{+}\left(x_{u}\right)} \backslash \mathcal{O}^{+}\left(x_{u}\right) \subset \partial \mathcal{O}^{+}\left(x_{u}\right)$. Thus $\mathcal{O}^{-}(y) \cap \mathcal{O}^{+}\left(x_{u}\right)=\varnothing$. On the other hand, $y$ is an equilibrium corresponding to a control in the interior of $\Omega$. Again Proposition 5.2 implies that $\mathcal{O}^{-}(y)$ is a neighborhood of $y$, and hence $\mathcal{O}^{-}(y) \cap \mathcal{O}^{+}\left(x_{u}\right) \neq \varnothing$. This contradiction shows that $\tau=1$ and $y=x_{v}$. Thus one can steer the system from any point $x_{u} \in \mathcal{C}$ to any other point $x_{v} \in \mathcal{C}$. It follows that $\mathcal{C}$ is contained in a single control set $D$. The same arguments show that, in fact, $\mathcal{C}$ is contained in the interior of $D$.

Remark 5.7 For scalar control, Theorem 4.3 shows that there are at most $n+1$ connected components of the set $E$ of equilibria, which consists of at most $n+1$ smooth curves. Thus also $E_{0}$ consists of at most $n+1$ smooth curves which, naturally, are pathwise connected. Hence, under the assumptions of Theorem 5.6, there are at most $n+1$ control sets containing an equilibrium in the interior.

In the rest of this section, we relate the controllability properties of system (1.1) to spectral properties of the matrices $A(u), u \in \Omega$.

Lemma 5.8 Consider the affine system (1.1) and suppose that $x_{u}$ is an equilibrium for a control value $u \in \operatorname{int}(\Omega)$ satisfying the rank condition (5.3).

(i) If every eigenvalue of $A(u)$ has negative real part, it follows that $\mathcal{O}^{-}\left(x_{u}\right)=\mathbb{R}^{n}$.

(ii) If every eigenvalue of $A(u)$ has positive real part, it follows that $\mathcal{O}^{+}\left(x_{u}\right)=\mathbb{R}^{n}$.

Proof By Proposition 5.2 the rank condition (5.3) implies that $\mathcal{O}^{-}\left(x_{u}\right)$ and $\mathcal{O}^{+}\left(x_{u}\right)$ are open.

(i) Let $0<\alpha<-\max \{\operatorname{Re} \lambda \mid \lambda$ an eigenvalue of $A(u)\}$. Then there is a constant $c_{0} \geq 1$ such that every solution of the autonomous linear differential equation $\dot{x}(t)=$ $A(u) x(t), x(0)=x_{0}$, satisfies

$$
\left\|e^{A(u) t} x_{0}\right\| \leq c_{0} e^{-\alpha t}\left\|x_{0}\right\| \text { for all } t \geq 0
$$

The variation-of-constants formula applied for $x \in \mathbb{R}^{n}$ and $x_{u}$ shows that 


$$
\begin{aligned}
& \varphi(t, x, u)-x_{u} \\
& \quad=e^{A(u) t} x+\int_{0}^{t} e^{A(u)(t-s)}[C u+d] d s-e^{A(u) t} x_{u}-\int_{0}^{t} e^{A(u)(t-s)}[C u+d] d s \\
& \quad=e^{A(u) t}\left(x-x_{u}\right) .
\end{aligned}
$$

Thus (5.7) implies

$$
\left\|\varphi(t, x, u)-x_{u}\right\| \leq c_{0} e^{-\alpha t}\left\|x-x_{u}\right\| \rightarrow 0 \text { for } t \rightarrow \infty .
$$

Since $\mathcal{O}^{-}\left(x_{u}\right)$ is a neighborhood of $x_{u}$, there exists $T>0$ such that $\varphi(T, x, u) \in$ $\mathcal{O}^{-}\left(x_{u}\right)$. Thus $x \in \mathcal{O}^{-}(\varphi(T, x, u)) \subset \mathcal{O}^{-}\left(x_{u}\right)$ and $\mathbb{R}^{n}=\mathcal{O}^{-}\left(x_{u}\right)$ follows.

(ii) For the system $\dot{x}(t)=-A(u) x-C u-d$, every eigenvalue of $-A(u)$ has negative real part. By (i) and time reversal, Lemma 2.2, the assertion follows.

Remark 5.9 An easy consequence of this lemma is that the system is controllable if there are $u, v \in \Omega$ with equilibria $x_{u}, x_{v}$ in the same pathwise connected subset of $E_{0}$ such that every eigenvalue of $A(u)$ has negative real part and every eigenvalue of $A(v)$ has positive real part; cf. Mohler [20, Main Result, p. 28] for the special case of inhomogeneous bilinear systems of the form (2.6).

The following corollary to Theorem 5.6 shows that there is a control set around the set of equilibria for uniformly hyperbolic matrices $A(u), u \in \Omega$.

Corollary 5.10 Consider an affine control system of the form (1.1) and assume that

(i) the control range $\Omega=\overline{\operatorname{int}(\Omega)}$ is compact and int $(\Omega)$ is pathwise connected;

(ii) the matrices $A(u)$ are uniformly hyperbolic in the following sense: There is $k$ with $0 \leq k \leq n$ such that for all $u \in \Omega$ there are $k$ eigenvalues with $\operatorname{Re} \lambda_{1}(u), \ldots$, $\operatorname{Re} \lambda_{k}(u)<0$ and $n-k$ eigenvalues with $\operatorname{Re} \lambda_{k+1}(u), \ldots, \operatorname{Re} \lambda_{n}(u)>0 ;$

(iii) every $u \in \operatorname{int}(\Omega)$ satisfies the rank condition (5.3).

Then the set $E=\overline{E_{0}}$ of equilibria is compact and connected, the set $E_{0}$ is pathwise connected, and there exists a control set $D$ with $E_{0} \subset \operatorname{int}(D)$.

Proof First observe that all matrices $A(u), u \in \Omega$, are invertible, since 0 is not an eigenvalue. Thus the set $E=\left\{x_{u} \mid u \in \Omega\right\}$ of equilibria is compact and $E_{0}$ is pathwise connected, since $x_{u}$ depends continuously on $u$. By Theorem 5.6 there exists a control set containing $E_{0}$ in the interior. Since pathwise connected sets are connected the set int $(\Omega)$ is connected, which implies that also $\Omega=\overline{\operatorname{int}(\Omega)}$ is connected, cf. Engelking [14, Corollary 6.1.11]. It also follows that the set $E=\overline{E_{0}}$ is connected.

If condition (ii) of Corollary 5.10 holds with $k=0$ or $k=n$, i.e., if all matrices $A(u)$ are stable or all are totally unstable, the rank condition (iii) for the linearized systems can be weakened.

Corollary 5.11 Let assumption (i) of Corollary 5.10 be satisfied and assume that there are at most finitely many points in int $(\Omega)$ such that the rank condition $(5.3)$ is violated. 
(i) If for all $u \in$ int $(\Omega)$ all eigenvalues of $A(u)$ have negative real parts, there exists a closed control set $D$ with $E_{0} \subset \operatorname{int}(D)$.

(ii) If for all $u \in$ int $(\Omega)$ all eigenvalues of $A(u)$ have positive real parts, there exists a control set $D$ with $E_{0} \subset \operatorname{int}(D)$.

Proof As in Corollary 5.10(i) it follows that the set $E_{0}$ of equilibria is pathwise connected. Consider equilibria $x_{u}, x_{v} \in E_{0}$ with $u, v \in$ int $(\Omega)$ and suppose that $x_{u}$ satisfies condition (5.3). Hence there is a control set $D_{u}$ containing $x_{u}$ in the interior. We use a construction similar to the one in the proof of Theorem 5.6: There is a continuous map $h:[0,1] \rightarrow E_{0}$ with $h(0)=x_{u}$ and $h(1)=x_{v}$. Let

$$
\tau:=\sup \left\{s \in[0,1] \mid \forall s^{\prime} \in[0, s]: h\left(s^{\prime}\right) \in D_{u}\right\}
$$

Observe that $\tau>0$, since $x_{u} \in$ int $\left(D_{u}\right)$. If $\tau<1$, then $y:=h(\tau) \in \partial D_{u}$ and $y=x_{w}$ is an equilibrium for some $w \in \operatorname{int}(\Omega)$. If $w$ satisfies (5.3), then by Proposition 5.5 $x_{w}$ is in the interior of a control set contradicting the choice of $\tau$. It remains to discuss the case where $w$ violates (5.3).

(i) Since all eigenvalues of $A(u)$ have negative real parts, Lemma 5.8(i) implies that $x_{w} \in \mathcal{O}^{-}\left(x_{u}\right)=\mathbb{R}^{n}$. Hence one can steer $x_{w}$ (in finite time) into the interior of $D_{u}$, and by continuous dependence on the initial value, this holds for all $x$ in a neighborhood $N\left(x_{w}\right)$. Note that $x_{w} \in \overline{D_{u}} \cap \partial D_{u}$. Since there are only finitely many points violating (5.3), all points $h\left(s^{\prime \prime}\right)$ with $s^{\prime \prime} \in(\tau, \tau+\varepsilon)$ for some $\varepsilon>0$ satisfy (5.3) and hence they are in a single control set $D^{\prime}$ and hence $x_{w} \in \overline{D^{\prime}}$. Then all points in the nonvoid intersection $N\left(x_{w}\right) \cap D^{\prime}$ can be steered into $D_{u}$. The same arguments show that one can steer points in $D_{u}$ into $D^{\prime}$, hence $D^{\prime}=D_{u}$. This contradicts the choice of $\tau$. It follows that $\tau=1$ and $x_{v} \in \overline{D_{u}}$. Using $x_{v} \in \mathcal{O}^{-}\left(x_{u}\right)=\mathbb{R}^{n}$ and $D_{u}=\overline{\mathcal{O}^{+}\left(x_{u}\right)} \cap \mathcal{O}^{-}\left(x_{u}\right)=\overline{\mathcal{O}^{+}\left(x_{u}\right)}$ one sees that $x_{v} \in D_{u}$. We conclude that all equilibria in $E_{0}$ are contained in the interior of a single closed control set.

(ii) Since all eigenvalues of $A(u)$ have positive real parts, Lemma 5.8(ii) implies that $x_{w} \in \mathcal{O}^{+}\left(x_{u}\right)=\mathbb{R}^{n}$. This shows that $x_{w}$ can be reached from $x_{u} \in \operatorname{int}\left(D_{u}\right)$. Continuous dependence on the initial value shows that all points in a neighborhood $N\left(x_{w}\right)$ of $x_{w}$ can be reached from the interior of $D_{u}$. Since there are only finitely many points violating (5.3), all points $h\left(s^{\prime \prime}\right)$ with $s^{\prime \prime} \in(\tau, \tau+\varepsilon)$ for some $\varepsilon>0$ are in a single control set $D^{\prime}$ and $x_{w} \in \overline{D^{\prime}}$. Then all points in the nonvoid intersection $N\left(x_{w}\right) \cap D^{\prime}$ can be reached from the interior of $D_{u}$. The same arguments show that some point in int $\left(D_{u}\right)$ can be reached from $D^{\prime}$, hence $D^{\prime}=D_{u}$. This contradicts the choice of $\tau$. It follows that $\tau=1$ and $x_{v} \in \overline{D_{u}}$. Using $x_{v} \in \mathcal{O}^{+}\left(x_{u}\right)=\mathbb{R}^{n}$ and $D_{u}=\overline{\mathcal{O}^{+}\left(x_{u}\right)} \cap \mathcal{O}^{-}\left(x_{u}\right)=\mathcal{O}^{-}\left(x_{u}\right)$ one sees that $x_{v} \in D_{u}$. We conclude that all equilibria in $E_{0}$ are contained in the interior of a single control set.

Remark 5.12 Remark 5.4 shows for an affine system of the form (1.1) with scalar control satisfying the assumptions of Proposition 5.3 that there are at most finitely many points $u$ where the rank condition (5.3) is violated.

Next we provide a sufficient condition for the existence of unbounded control sets. 
Theorem 5.13 Consider an affine control system of the form (1.1), let $\mathcal{C}$ be a pathwise connected subset of the set $E_{0}$ of equilibria of system (1.1) and define $\Omega(\mathcal{C})=\{u \in$ $\left.\operatorname{int}(\Omega) \mid x_{u} \in \mathcal{C}\right\}$. Assume that

(i) there is $u^{0} \in \overline{\Omega(\mathcal{C})}$ such that $A\left(u^{0}\right)$ has the eigenvalue $\lambda_{0}=0$ and $C u^{0}+d$ is not in the range of $A\left(u^{0}\right)$;

(ii) every $u \in \Omega(\mathcal{C}), u \neq u^{0}$, satisfies $\operatorname{rank} A(u)=n$ and the rank condition (5.3).

Then, there is an unbounded control set $D \subset \mathbb{R}^{n}$ containing $\mathcal{C}$ in the interior. More precisely, for $u^{k} \in \Omega(\mathcal{C})$ with $u^{k} \rightarrow u^{0}$ for $k \rightarrow \infty$, the equilibria $x_{u^{k}} \in \mathcal{C} \subset \operatorname{int}(D)$ satisfy for $k \rightarrow \infty$

$$
\left\|x_{u^{k}}\right\| \rightarrow \infty \text { and } \frac{x_{u^{k}}}{\left\|x_{u^{k}}\right\|} \rightarrow \operatorname{ker} A\left(u^{0}\right) \cap \mathbb{S}^{n-1} .
$$

Proof By Theorem 5.6 there is a control set $D$ containing $\mathcal{C}$ in the interior. In order to show that $D$ is unbounded, we argue similarly as in the scalar situation in Theorem 4.3 .

Let $u^{k} \in \Omega(\mathcal{C})$ converge to $u^{0}$ and assume, by way of contradiction, that $x_{u^{k}}$ remains bounded, hence we may suppose that there is $x^{0} \in \mathbb{R}^{n}$ with $x_{u^{k}} \rightarrow x^{0}$. Then the equalities

$$
A\left(u^{k}\right) x_{u^{k}}=-\left[C u^{k}+d\right]
$$

lead for $k \rightarrow \infty$ to

$$
A\left(u^{0}\right) x_{u^{0}}=-\left[C u^{0}+d\right]
$$

contradicting assumption (i). We have shown that $x_{u^{k}}$ becomes unbounded for $k \rightarrow \infty$. Since $C u^{k}+d \rightarrow C u^{0}+d$, we get

$$
A\left(u^{k}\right) \frac{x_{u^{k}}}{\left\|x_{u^{k}}\right\|}=\frac{1}{\left\|x_{u^{k}}\right\|}\left(C u^{k}+d\right) \rightarrow 0 .
$$

On the other hand, every cluster point $y \in \mathbb{R}^{n}$ of the bounded sequence $\frac{x_{u^{k}}}{\left\|x_{u^{k}}\right\|}$ satisfies $\|y\|=1$ and (5.8) follows.

Theorem 5.13 sheds some light on the relation between controllability properties of affine systems and their homogeneous bilinear parts: By Theorem 3.15 assumption (i) is related to the existence of a control set of the latter system in $\mathbb{R}^{n}$.

We state the following result concerning closed invariant cones (cf. Remark 3.17). This is formulated in the context of semigroup actions. Denote by $S_{\text {aff }}$ and $S_{\text {hom }}$ the system semigroups of the affine and the homogeneous bilinear control systems given by (1.1) and (2.7), respectively. They correspond to piecewise constant controls (see Appendix A of [10]). The system group of the affine control system is given by the semidirect product $G=H \rtimes \mathbb{R}^{n}$, where $H$ is the system group of the homogenous 
bilinear system. The affine group operation is defined by $(g, v) \cdot(h, w)=(g h, v+g w)$ for all $(g, v),(h, w) \in G$, and the affine action of $G$ on $\mathbb{R}^{n}$ is given by $(g, v) \cdot w=$ $g w+v$ with $(g, v) \in G$ and $w \in \mathbb{R}^{n}$ using the linear action of $H$ on $\mathbb{R}^{n}$. A set $Q \subset \mathbb{R}^{n}$ is invariant under $S_{\text {aff }}$ and $S_{\text {hom }}$ if and only if it is invariant for the affine control system and the homogeneous bilinear control systems, respectively. We get the following relations between invariance of a closed cone for $S_{\text {aff }}$ and $S_{\text {hom }}$.

Proposition 5.14 Consider an affine control system of the form (1.1) and its homogeneous bilinear part (2.7), and let $K$ be a closed cone in $\mathbb{R}^{n}$.

(i) Suppose that $K$ is invariant for the homogeneous bilinear part and $C u+d \in K$ for all $u \in \Omega$. Then $K$ is invariant for the affine control system.

(ii) If $K$ is invariant for the affine control system, then it is invariant for the homogeneous bilinear part.

Proof Assertion (i) is immediate from the definitions. The assumption in (ii) means that $(g, v) \cdot w \in K$ for all $(g, v) \in S_{\text {aff }}$ and $w \in K$. Suppose, by way of contradiction, that there exists $g \in S_{\text {hom }}$ with $x:=g w \notin K$ for some $w \in K$; hence $g(\lambda w)=$ $\lambda(g w)=\lambda x \notin K$ for all $\lambda>0$. It follows that

$$
\inf \left\{\left\|\lambda x-\lambda w^{\prime}\right\| \mid w^{\prime} \in K\right\}=\lambda \inf \left\{\left\|x-w^{\prime}\right\| \mid w^{\prime} \in K\right\} \rightarrow \infty \text { for } \lambda \rightarrow \infty .
$$

Hence for every $v \in \mathbb{R}^{n}$ there is $\lambda>0$ such that inf $\left\{\left\|g(\lambda w)+v-w^{\prime}\right\| \mid w^{\prime} \in K\right\}>0$ implying $g(\lambda w)+v \notin K$. This means for the action of $S_{\text {aff }}$ that $(g, v) \cdot(\lambda w)=$ $g(\lambda w)+v \notin K$ contradicting the invariance of $K$ for $S_{\text {aff }}$.

Remark 5.15 Jurdjevic and Sallet [17, Theorem 2] shows that controllability of an affine control system without fixed points can be guaranteed if its homogeneous bilinear part is controllable. Furthermore, for $Q \subset \mathbb{R}^{n}$ let $\mathrm{A}(Q)$ be its affine hull. Suppose that $Q$ is invariant for the affine control system. Then [17, Lemma 3] implies that $\mathrm{A}(Q)$ is invariant for the affine control system and the set $\left\{\sum_{i=1}^{p} \lambda_{i} q_{i} \mid q_{i} \in Q, \lambda_{i} \in \mathbb{R}\right.$ with $\left.\sum_{i=1}^{p} \lambda_{i}=0, p \in \mathbb{N}\right\}$ is invariant for its homogeneous bilinear part.

Finally, we illustrate Theorem 5.6 and Theorem 5.13 by discussing the control sets for two affine systems. Recall that by Theorem 3.15, the existence of a control $u^{0} \in \operatorname{int}(\Omega)$ such that 0 is an eigenvalue of $A\left(u^{0}\right)$ is connected with the existence of an unbounded control set of the bilinear system $\dot{x}=A(u) x$.

Example 5.16 Consider again Example 4.4. In order to describe the control sets we first check the controllability rank condition (5.3) for $|u| \neq \frac{1}{2}$. By (4.4)

$$
B^{\prime}(u)=C+B x_{u}=\left[\begin{array}{l}
0 \\
1
\end{array}\right]+\frac{u}{4 u^{2}-1}\left[\begin{array}{ll}
2 & 0 \\
0 & 2
\end{array}\right]\left[\begin{array}{c}
1 \\
-2 u
\end{array}\right]=\frac{1}{4 u^{2}-1}\left[\begin{array}{c}
2 u \\
-1
\end{array}\right],
$$

and hence

$$
\left(4 u^{2}-1\right)\left[B^{\prime}(u), A(u) B^{\prime}(u)\right]=\left[\begin{array}{c}
2 u \\
-1
\end{array},\left(\begin{array}{cc}
2 u & 1 \\
1 & 2 u
\end{array}\right)\left(\begin{array}{c}
2 u \\
-1
\end{array}\right)\right]=\left[\begin{array}{cc}
2 u & 4 u^{2}-1 \\
-1 & 0
\end{array}\right] .
$$


Thus the rank condition (5.3) holds in every equilibrium $\left(x_{u}, y_{u}\right)$ with $|u| \neq \frac{1}{2}$.

Next we discuss the control sets for several control ranges given by a compact interval.

- Let $\Omega=\left[u_{*}, u^{*}\right]$ with $\frac{1}{2}<u_{*}<u^{*}$. Then the set of equilibria is given by the compact subset $\left\{\left(x_{u}, y_{u}\right) \mid u \in\left[u_{*}, u^{*}\right]\right\} \subset \mathcal{B}_{3}$. By Theorem 5.6 there is a single control set $D_{3}$ with $\left(x_{u}, y_{u}\right) \in \operatorname{int}\left(D_{3}\right)$ for all $u \in\left(u_{*}, u^{*}\right)$.

- Let $\Omega=\left[u_{*}, u^{*}\right]$ with $u_{*}<u^{*}<-\frac{1}{2}$. Then the set of equilibria is given by the compact subset $\left\{\left(x_{u}, y_{u}\right) \mid u \in\left[u_{*}, u^{*}\right]\right\} \subset \mathcal{B}_{2}$. By Theorem 5.6 there is a single closed control set $D_{2}$ with $\left(x_{u}, y_{u}\right) \in \operatorname{int}\left(D_{2}\right)$ for all $u \in\left(u_{*}, u^{*}\right)$.

- Let $\Omega=\left[u_{*}, u^{*}\right]$ with $-\frac{1}{2}<u_{*}<u^{*}<\frac{1}{2}$. Then the set of equilibria is given by the compact subset $\left\{\left(x_{u}, y_{u}\right) \mid u \in\left[u_{*}, u^{*}\right]\right\} \subset \mathcal{B}_{1}$. By Theorem 5.6 there is a single control set $D_{1}$ with $\left(x_{u}, y_{u}\right) \in \operatorname{int}\left(D_{1}\right)$ for all $u \in\left(u_{*}, u^{*}\right)$.

- Let $\Omega=[-1,1]$. Then the connected components of the set $E_{0}$ of equilibria are

$$
\begin{aligned}
& \mathcal{C}_{1}=\left\{\left(x_{u}, y_{u}\right) \mid u \in\left(-\frac{1}{2}, \frac{1}{2}\right)\right\}, \quad \mathcal{C}_{2}=\left\{\left(x_{u}, y_{u}\right) \mid u \in\left(-1,-\frac{1}{2}\right)\right\}, \\
& \mathcal{C}_{3}=\left\{\left(x_{u}, y_{u}\right) \mid u \in\left(\frac{1}{2}, 1\right)\right\},
\end{aligned}
$$

and there are control sets $D_{i}$ with $\mathcal{C}_{i} \subset \operatorname{int}\left(D_{i}\right)$ for $i=1,2,3$. Since these sets of equilibria are unbounded also the control sets are unbounded. Based on Proposition 4.1, a lengthy argument involving the phase portraits for constant controls shows that one cannot steer the system from $D_{2}$ to $D_{3}$ or $D_{1}$ and from $D_{1}$ to $D_{3}$, hence these control sets are pairwise different.

Next we take up the linear oscillator from Example 3.20 and consider an associated affine control system. We will show that there are two unbounded control sets.

Example 5.17 Consider the affine control system given by

$$
\ddot{x}+3 \dot{x}+(1+u(t)) x=u(t)+d \text { with } u(t) \in[-\rho, \rho],
$$

where $\rho \in\left(1, \frac{5}{4}\right)$ and $d \in \mathbb{R}$. Hence the system equation has the form

$$
\left[\begin{array}{l}
\dot{x} \\
\dot{y}
\end{array}\right]=\left[\begin{array}{cc}
0 & 1 \\
-1 & -3
\end{array}\right]\left[\begin{array}{l}
x \\
y
\end{array}\right]+u(t)\left[\begin{array}{cc}
0 & 0 \\
-1 & 0
\end{array}\right]\left[\begin{array}{l}
x \\
y
\end{array}\right]+u(t)\left[\begin{array}{l}
0 \\
1
\end{array}\right]+\left[\begin{array}{l}
0 \\
d
\end{array}\right] .
$$

For the equilibria with $u \neq-1$ we find

$$
\left[\begin{array}{l}
x_{u} \\
y_{u}
\end{array}\right]=-\left[\begin{array}{cc}
0 & 1 \\
-1-u-3
\end{array}\right]^{-1}\left[\begin{array}{c}
0 \\
u+d
\end{array}\right]=\left[\begin{array}{cc}
\frac{3}{1+u} & \frac{1}{1+u} \\
-1 & 0
\end{array}\right]\left[\begin{array}{c}
0 \\
u+d
\end{array}\right]=\left[\begin{array}{c}
\frac{d+u}{1+u} \\
0
\end{array}\right]
$$

This yields that the connected components of the set $E_{0}$ of equilibria are

$$
\mathcal{C}_{1}=\left\{\left[\begin{array}{c}
\frac{d+u}{1+u} \\
0
\end{array}\right] \mid u \in(-\rho,-1)\right\}, \quad \mathcal{C}_{2}=\left\{\left[\begin{array}{c}
\frac{d+u}{1+u} \\
0
\end{array}\right] \mid u \in(-1, \rho)\right\} .
$$


For $d=1$ there is a single equilibrium given by $\left(x_{u}, y_{u}\right)^{\top}=(1,0)^{\top}$ for every $u \neq-1$. Henceforth we assume $d \neq 1$.

Let $d<1$. Then for $u \in[-\rho,-1)$ one obtains $d+u<1+u<0$, and for $u \in(-1, \rho]$ one obtains $1+u>0$, hence

$$
\mathcal{C}_{1}=\left\{\left[\begin{array}{l}
x \\
0
\end{array}\right] \mid x \in\left(\frac{d-\rho}{1-\rho}, \infty\right)\right\}, \quad \mathcal{C}_{2}=\left\{\left[\begin{array}{l}
x \\
0
\end{array}\right] \mid x \in\left(-\infty, \frac{d+\rho}{1+\rho}\right)\right\} .
$$

Let $d>1$. Then $u \in[-\rho,-1)$ yields $1+u<0$ and $u \in(-1, \rho]$ yields $1+u>0$, hence

$$
\mathcal{C}_{1}=\left\{\left[\begin{array}{l}
x \\
0
\end{array}\right] \mid x \in\left(-\infty, \frac{d-\rho}{1-\rho}\right)\right\}, \quad \mathcal{C}_{2}=\left\{\left[\begin{array}{l}
x \\
0
\end{array}\right] \mid x \in\left(\frac{d+\rho}{1+\rho}, \infty\right)\right\} .
$$

Note that $\mathcal{C}_{1} \cap \mathcal{C}_{2}=\varnothing$ for all $d$. The equilibria in $\mathcal{C}_{1}$ are hyperbolic, since here $\lambda_{1}(u)<0<\lambda_{2}(u)$ with $\lambda_{2}(u) \rightarrow 0$ for $u \rightarrow-1$. The equilibria in $\mathcal{C}_{2}$ are stable nodes since here $\lambda_{1}(u)<\lambda_{2}(u)<0$.

Next we check the assumptions of Theorem 5.13. For $u^{0}=-1$ the matrix $A(-1)=$ $\left[\begin{array}{cc}0 & 1 \\ 0 & -3\end{array}\right]$ has the eigenvalue $\lambda_{0}=0$ with eigenspace $\mathbb{R} \times\{0\}$, and $\operatorname{Im} A(-1)=$ $\{(y,-3 y) \mid y \in \mathbb{R}\}$. Furthermore

$$
C u^{0}+d=\left[\begin{array}{l}
0 \\
1
\end{array}\right](-1)+\left[\begin{array}{l}
0 \\
d
\end{array}\right]=\left[\begin{array}{c}
0 \\
d-1
\end{array}\right]
$$

is not in the range of $A(-1)$. This verifies assumption (i) in Theorem 5.13. In order to check the rank condition (5.3) we compute for $u \neq-1$

$$
\begin{aligned}
B^{\prime}(u) & =C+B\left[\begin{array}{l}
x_{u} \\
y_{u}
\end{array}\right]=\left[\begin{array}{l}
0 \\
1
\end{array}\right]+\left[\begin{array}{cc}
0 & 0 \\
-1 & 0
\end{array}\right]\left[\begin{array}{c}
\frac{d+u}{1+u} \\
0
\end{array}\right]=\left[\begin{array}{c}
0 \\
\frac{1-d}{1+u}
\end{array}\right], \\
A(u) B^{\prime}(u) & =\left[\begin{array}{cc}
0 & 1 \\
-1-u-3
\end{array}\right]\left[\begin{array}{c}
0 \\
\frac{1-d}{1+u}
\end{array}\right]=\left[\begin{array}{c}
\frac{1-d}{1+u} \\
-3 \frac{1-d}{1+u}
\end{array}\right] .
\end{aligned}
$$

Hence $\operatorname{rank}\left[B^{\prime}(u), A(u) B^{\prime}(u)\right]=2$ for $u \neq-1$. Theorem 5.13 implies that there are unbounded control sets $D_{i}$ containing the equilibria in $\mathcal{C}_{i}, i=1,2$, in the interior. For $u^{k} \rightarrow u^{0}=-1$, the equilibria $\left(x_{u^{k}}, y_{u^{k}}\right)=\left(x_{u^{k}}, 0\right)$ become unbounded for $k \rightarrow \infty$ and

$$
\frac{\left(x_{u^{k}}, 0\right)}{\left\|\left(x_{u^{k}}, 0\right)\right\|} \in \operatorname{ker} A(-1) \cap \mathbb{S}^{1}=\left\{\left[\begin{array}{l}
1 \\
0
\end{array}\right],\left[\begin{array}{c}
-1 \\
0
\end{array}\right]\right\} \text { for all } k
$$

In the simple case considered here, the latter assertion is already clear by formula (5.9) for the equilibria.

While the asymptotic stability of the equilibria in $\mathcal{C}_{2}$ implies that one can steer the system from $\mathcal{C}_{1}$ to $\mathcal{C}_{2}$, the converse does not hold which follows by inspection of the phase portraits for the controls in $[-\rho,-1]$ and $[-1, \rho]$. It follows that $D_{1} \neq D_{2}$. 
Acknowledgements We would like to thank two anonymous reviewers whose comments helped to improve the paper.

Funding Open Access funding enabled and organized by Projekt DEAL.

Open Access This article is licensed under a Creative Commons Attribution 4.0 International License, which permits use, sharing, adaptation, distribution and reproduction in any medium or format, as long as you give appropriate credit to the original author(s) and the source, provide a link to the Creative Commons licence, and indicate if changes were made. The images or other third party material in this article are included in the article's Creative Commons licence, unless indicated otherwise in a credit line to the material. If material is not included in the article's Creative Commons licence and your intended use is not permitted by statutory regulation or exceeds the permitted use, you will need to obtain permission directly from the copyright holder. To view a copy of this licence, visit http://creativecommons.org/licenses/by/4.0/.

\section{References}

1. Ayala V, Cruz E, Kliemann W, Laura-Guarachi LR (2016) Controllability properties of bilinear systems in dimension 2. J Math Comput Sci 16:554-575

2. Bacciotti A, Vivalda J-C (2013) On radial and directional controllability of bilinear systems. Systems Control Lett 62(7):575-580

3. Bonnard B (1981) Contrôllabilité des systèmes bilinéaires. Math Syst Theory 15:79-92

4. Bonnard B, Jurdjevic V, Kupka I, Sallet G (1982) Transitivity of families of invariant vector fields on the semi-direct product of Lie groups. Trans Am Math Soc 271:525-535

5. Boothby W, Wilson EN (1979) Determination of transitivity of bilinear systems. SIAM J Control Optim 17:212-221

6. Braga Barros CJ, San Martin LAB (1996) On the number of control sets on projective spaces. Syst Control Lett 29:21-26

7. Cannarsa D, Sigalotti M (2021) Approximately controllable finite-dimensional bilinear systems are controllable. Syst Control Lett 157 Article 105028

8. Cassels JWS (1957) Introduction to Diophantine Approximations. Cambridge University Press

9. Colonius F, Kliemann W (1995) Asymptotic null controllability of bilinear systems. In: Jakubczyk B, Respondek W (eds) Nonlinear control and differential inclusions, vol 32. Banach Center Publications, Warsaw, pp. 139-148

10. Colonius F, Kliemann W (2000) The dynamics of control. Birkhäuser

11. Do Rocio O, San Martin LAB, Santana AJ (2006) Invariant cones and convex sets for bilinear control systems and parabolic type of semigroups. J Dyn Control Syst 12(3):419-432

12. Do Rocio O, Santana AJ, Verdi M (2009) Semigroups of affine groups, controllability of affine systems and affine bilinear systems in $\operatorname{Sl}(2, \mathbb{R}) \rtimes \mathbb{R}^{2}$. SIAM J Control Optim 48(2):1080-1088

13. Elliott DL (2008) Bilinear control systems, matrices in action. Kluwer Academic Publishers

14. Engelking R (1977) General topology. PWN - Polish Scientific Publishers, Warszawa

15. Gauthier JP, Bornard G (1982) Controllabilité des systèmes bilinéaires. SIAM J Control Optim 20(3):377-384

16. Jurdjevic V, Kupka I (1981) Control systems on semi-simple Lie groups and their homogeneous spaces. Annales de l'institut Fourier, tome 31(4):151-179

17. Jurdjevic V, Sallet G (1984) Controllability properties of affine systems. SIAM J Control Optim 22(3):501-508

18. Jurdjevic V (1997) Geometric control theory. Cambridge University Press

19. Lee EB, Markus L (1986) Foundations of optimal control theory, Robert E. Krieger Publishing Company, Original Edition 1967 Reprint Edition 1986 with corrections

20. Mohler RR (1973) Bilinear control processes. Academic Press, New York and London

21. Rink RE, Mohler RR (1968) Completely controllable bilinear systems. SIAM J Control Optim 6(3):477-486

22. Sachkov YuL (1998) On invariant orthants of bilinear systems. J Dyn Control Syst 4(1):137-147

23. San Martin L (1993) Invariant control sets on flag manifolds. Math Control Signals Syst 6:41-61

24. Sontag E (1998) Mathematical control theory. Springer 
25. Tchebychef PL (1899) Sur une question arithmétique. Oeuvres Tome I. Imprimerie de l'Academie Impériale des Sciences, St. Petersburg, pp 639-684

26. Teschl G (2012) Ordinary Differential Equations and Dynamical Systems, Graduate Studies in Math, vol 149. Amer Math Soc

Publisher's Note Springer Nature remains neutral with regard to jurisdictional claims in published maps and institutional affiliations. 